\title{
On the time-consistent stochastic dominance risk averse measure for tactical supply chain planning under uncertainty
}

\author{
Laureano F. Escudero ${ }^{\mathrm{a}, *}$, Juan Francisco Monge ${ }^{\mathrm{b}}$, Dolores Romero Morales ${ }^{\mathrm{c}}$ \\ ${ }^{a}$ Estadística e Investigación Operativa,Universidad Rey Juan Carlos, Móstoles (Madrid), Spain \\ ${ }^{\mathrm{b}}$ Centro de Investigación Operativa,Universidad Miguel Hernández, Elche (Alicante), Spain \\ ${ }^{\mathrm{c}}$ Copenhagen Business School,Frederiksberg, Denmark
}

\section{A R T I C L E I N F O}

\section{Article history:}

Received 21 February 2016

Revised 23 February 2017

Accepted 13 July 2017

Available online $\mathrm{xxx}$

\section{Keywords:}

Tactical supply chain planning

Nonlinear separable objective function

Multistage stochastic integer optimization

Risk management

Time-consistency

Stochastic nested decomposition

\begin{abstract}
A B S T R A C T
In this work a modeling framework and a solution approach have been presented for a multi-period stochastic mixed 0-1 problem arising in tactical supply chain planning (TSCP). A multistage scenario tree based scheme is used to represent the parameters' uncertainty and develop the related Deterministic Equivalent Model. A cost risk reduction is performed by using a new time-consistent risk averse measure. Given the dimensions of this problem in real-life applications, a decomposition approach is proposed. It is based on stochastic dynamic programming (SDP). The computational experience is twofold, a comparison is performed between the plain use of a current state-of-the-art mixed integer optimization solver and the proposed SDP decomposition approach considering the risk neutral version of the model as the subject for the benchmarking. The add-value of the new risk averse strategy is confirmed by the computational results that are obtained using SDP for both versions of the TSCP model, namely, risk neutral and risk averse.
\end{abstract}

(c) 2017 Elsevier Ltd. All rights reserved.

\section{Introduction}

1.1. The problem to be addressed, its importance and difficulty to solve

The deterministic version of the tactical supply chain planning problem (TSCP) that is discussed in this work is based on the reallife case in the assembly sector. It has a broad applicability, specifically, in sectors such as car, computer and domestic appliances manufacturing, among others. It is the case in which a company with multiple raw material suppliers, plants, products, tiers of production in the bill of material (BoM) and markets needs to satisfy a product demand vector over a given time horizon. The goal is to determine a raw material supplying plan and a master production, inventory and distribution planning that best makes use of the available resources and their capacity extension acquisitions in the whole supply chain for each period of a given time horizon.

th This research has been partially supported by the projects MTM2015-63710-P (L.F. Escudero) and MTM2016-79765-P (Juan F. Monge) from the Spanish Ministry of Economy, Industry and Competitiveness and the European Regional Development Fund (AEI/FEDER, UE).

* Corresponding author.

E-mail addresses: laureano.escudero@urjc.es (L.F. Escudero), monge@umh.es (J.F. Monge),drm.eco@cbs.dk (D.R. Morales).
The resources' best use consists of minimizing the raw material supplying commitment cost, the production and inventory costs in the plants, and the product backlog and demand lost penalization along the time horizon. The raw material supplying commitment cost is frequently modeled by a piecewise linear, concave and nondecreasing function of the total volume to commit for the whole time horizon. Typical types of constraints (some of them related to either-or decisions) are as follows: Balance equations of endproducts and components, conditional lower and upper bounds for raw material supplying and product release, resource consumption bounds and capacity extension acquisitions, and balance equations of lost demand and backlogging, among others. There are different types of resources at different levels for groups of consecutive periods (so-called stages) along the time horizon. The cost of the resources' capacity extension acquisition is expressed as a piecewise discrete and nondecreasing function. Another important feature of the problem is that that no warehouses are encouraged for intermediate components and products stocking, although some stocking is allowed in the plants. Even the burden of raw material stocking is frequently transferred to the suppliers.

There are many contributions on TSCP for several variants to the case presented above, see the seminal works Cohen and Lee (1989), Escudero (1994) and Shapiro (1993), among others. However, given the volatility in the markets and the dynamic nature of the planning problem, some parameters, besides being not known 
with certainty when decisions are to be made, have high variability in their realizations. Those parameters are the coefficients in the function to minimize (e.g., production cost), the right-handside vector (rhs) of some of the constraints (e.g., product demand and resource availability) and the constraint matrix (e.g., product demand lost fraction). Usually, those uncertain parameters are represented by their expected value (EV) and, thus, the parameters' variability is not considered in the model. Hence, the very popular EV strategy is frequently inadequate for problem solving. This work deals with stochastic tactical supply chain planning (STSCP), where the available information about the parameters' uncertainty is represented in the form of a finite set of scenarios. They are considered in the constraints of the model and the expected cost to be minimized. The problem's formulation is so-called deterministic equivalent model (DEM), see in e.g., Birge and Louveaux (2011) the main concepts on stochastic optimization. An important uncertain parameter is the lost demand fraction for the products in each period of the time horizon. Given the high competitive character of the markets, the demand volatility and the potential unavailability of required resources, by no means the non-served demand in a period can be considered with certainty a backlog for the next one. So, the lost demand fraction is uncertain. On the other hand, the time latency in the availability of raw material and subassemblies for production and end-product availability in the markers should be taken into account, due to its strong implication on the related multistage scenario tree.

\subsection{Current topics in stochastic optimization}

In the presence of uncertain parameters, different approaches for solving nonlinear separable mixed $0-1$ problems can be found in the literature in the two-stage and multistage settings. A recent review of decomposition algorithms is presented in Aldasoro et al. (2017), most of the algorithms are intended for problem solving with moderate model dimensions. For bigger instances, some types of scenario cluster decomposition approaches can be used, such as Branch-and-Fix Coordination (Aldasoro et al., 2017; AlonsoAyuso et al., 2003), two-stage Lagrangean decomposition (Carœand Schultz, 1999), Progressive Hedging algorithm (Gade et al., 2016) and multistage cluster Lagrangean decomposition (Escudero et al., 2016b), among others. For instances with very large dimensions, such as real-life STSCP instances, matheuristic approaches should be used, as the algorithms that belong to the stochastic nested decomposition methodology, see Aldasoro et al. (2015), Cristobal et al. (2009), Escudero et al. (2015) and Zou etal. (2016). Those stochastic optimization approaches deal with the minimization of the objective function expected value alone, so-called risk neutral (RN) strategies. However, the variability of the STSCP cost over the scenarios is not completely taken into account and, then, inducing a negative cost impact of the RN solutions on low-probability highcost scenarios.

Some approaches that present risk reduction measures are as follows: scenario immunization, see Dembo (1991) and its treatment in Escudero (1995), semi-deviations (Ahmed, 2006; Ogryczak and Ruszczyński, 1999), min-risk (i.e, excess probabilities) (Ahmed, 2006; Schultz and Tiedemann, 2003), value-and-risk (Gaivoronski and Plug, 2005), conditional value-at-risk (CVaR) (Ahmed, 2006; Pflug and Pichler, 2015b; Rockafellar and Uryasev, 2000; Schultz and Tiedemann, 2006) and stochastic dominance (SD) strategies (Escudero etal., 2016a; Gollmer etal., 2011; 2008). For computational comparison of the time inconsistent versions of those measures, see Alonso-Ayuso et al. (2014).

Recent state-of-the-art surveys on risk management, specifically dealing with supply chains, can be found in Esmaeilikia (2013), Esmaeilikia et al. (2016a); 2016b), Fahimnia et al. (2015), Heckmann etal. (2015) and Ho etal. (2015). However, there are only a few re- cent STSCP works dealing with risk averse measures, mainly CVaR in Alem and Morabito (2013) and Nickel et al. (2012), and meanrisk and minmax for robust solutions in Govindan and Fattahi (2017) presenting real-life cases for validating the proposed approaches, among others.

In addition, most of the approaches presented above are targeting stochastic two-stage problems and specific decomposition algorithms are developed. In a stochastic multi-period two-stage problem, a node in the scenario tree has only one immediate successor and, then, its parameters can only influence on the parameters of the same scenario. So, the non-anticipativity (NA) principle is not satisfied for the variables in any node but the first one.

However, in a multistage setting the realizations in any node in the scenario tree (besides the first one) may have an influence on the parameters of its successor nodes in different scenarios. So, the nodes that belong to any stage (but the first one) have a conditional probability based on their ancestor common node (i.e., it is a Markovian process). Hence, the NA principle should be satisfied for the decision variables of the nodes in the tree with a one-toone correspondence with the group of scenarios that have identical realizations up to the node-related period.

Additionally, a SD risk averse measure is very appropriate for the STSCP cost risk reduction, since it controls the expected cost excess over a set of modeler-driven increasingly cost thresholds in the scenarios. That control is materialized in a policy for which the higher the cost threshold is, the smaller the modeler-driven upper bounds should be for the expected cost excess over the threshold and the failure probability to satisfy it.

\subsection{Main contributions of this work in STSCP problem solving}

The main contributions of our work are aimed to reducing some gaps in the literature on STSCP problem solving that have been identified above. They are as follows:

1. Presenting a realistic multi-period STSCP problem with a multitier BoM for a multi-product, multi-supplier and multi-market setting, a piecewise linear objective function and uncertainty in dynamic product demand, demand lost fraction, production cost and available resource base capacity.

2. Modeling the STSCP via scenario analysis by using a multiperiod mixed 0-1 full recourse Deterministic Equivalent Model (DEM) with S2 sets to represent the piecewise linear terms in the objective function. Because the supplying ordering time lag and transportation time between vendors and plants, origin and destination plants, and end-product plants and markets, the ordering and delivering could belong to different time periods. As a consequence, different modeling objects are required, given the variety of scenarios that may occur in the time interval between the ordering and the delivering.

3. Modeling the risk reduction of the cost impact of the STSCP solutions on low-probability high-cost scenarios (i.e., the black swans). For the reasons that will be fully formalized in Section 4, the dynamic SD risk averse measure takes benefit from a new version so-called expected conditional stochastic dominance (ECSD), that is introduced in this work. It is a timeconsistent risk averse measure consisting of a mixture of firstand second-order SD functionals related to a set of modelerdriven profiles in the nodes of a given subset of periods. Each profile is included by a cost threshold, a bound on the expected cost excess over the threshold in the scenario group with a one-to-one correspondence with the nodes of the selected periods, and a bound on the probability of any of those scenarios to fail on satisfying the cost threshold. The rationale behind it is that the solution of any node and its successor node set in the scenario tree should not consider the data, constraints and 


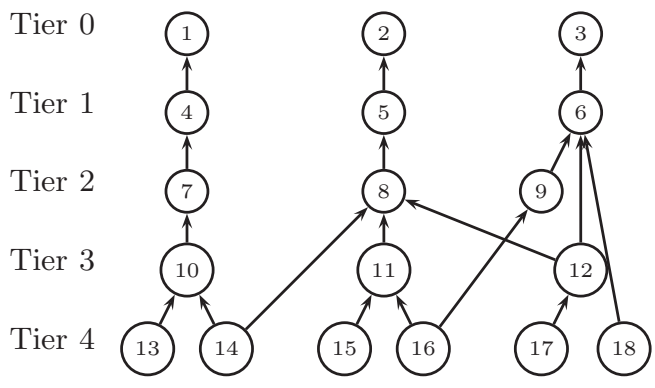

Fig. 1. Bill of material in a supply chain.

variables that belong to scenarios that cannot occur from that point. So, ECSD belongs to the type of risk averse measures that have the time consistency property as presented in Homem-de Mello and Pagnoncelli (2016).

4. Introducing a specialization of our stochastic nested decomposition (SND) approach in Cristobal et al. (2009) based on stochastic dynamic programming (SDP) for dealing with the proposed time-consistent risk averse measure, given the high number of cross-scenario constraints and, as a consequence, the nice scenario node-based modeling structure is lost.

5. Performing a computational comparison in the multistage setting between the time-consistent ECSD cost risk reduction strategy and the Risk Neutral (RN) one, and between the timeconsistent model and its time-inconsistent counterpart. Additionally, the comparison is also performed between the plain use of a state-of-the-art mixed integer optimization solver of choice and our matheuristic SDP specialization for STSCP solving.

The remainder of the paper is organized as follows. Section 2 introduces the TSCP problem and the deterministic data is presented. Section 3 presents the uncertain information as well as the RN based model for STSCP. Section 4 deals with the timeinconsistent version of the stochastic dominance risk averse measure, so-called TSD, and it introduces the time-consistent ECSD version. Section 5 introduces the SDP algorithm to deal with the ECSD measure for STSCP problem solving. Section 6 reports the main results of our computational experience. Finally, some conclusions are drawn from this work in Section 7 and it also outlines the future research.

\section{The TSCP problem}

The TSCP problem of concern is presented in detail. It is based on a real life case but, in fact, it has a broad applicability. For the sake of presentation, let us fix some notation to be used throughout the paper. The problem is formalized next.

A time horizon is a set of consecutive time periods, which do not necessarily have the same length, where the planning is considered. The periods are grouped in the so-called stages.

A product is any item whose production volume, location and scheduling is decided by the planner. An end-product is a final output of the supply chain. A subassembly is a product that is assembled by the supply chain and is used to produce either an end-product or another subassembly. Therefore, the term product refers to both end-products and subassemblies. A component is any storable item that is required for the production.

A BoM of a product is the structuring of the set of components that are required for its manufacturing/assembly, see Fig. 1. The BoM can be described as a set of tiers in the supply chain. A so-called first tier component of a product is a component that is directly required for the manufacturing/assembly of that product. Note that a component can be used for the manufactur-

ing/assembly of one or more products. The cycle time of a product is the set of consecutive time periods that are required for its completion from its release in the manufacturing/assembly line until its availability for use.

A raw material is any storable item that is required to produce any of the products, but whose own BoM is not a concern to the planner. Therefore, subassemblies and raw materials are components. The stock of an item (either a product or a raw material) is its available volume at the end of a given time period. A resource is any non-storable item that is required for the manufacturing/assembly of products and its availability is limited for any period along the time horizon, although its base capacity can be expanded.

A vendor is any external source supplying raw materials. It is assumed that each raw material is only supplied by one vendor. We model the supplying cost for a given raw material as a piecewise linear, concave and nondecreasing function. The supplying volume commitment is performed at the beginning of the time horizon, and a reward is received for each unit in surplus at the end of the time horizon. We assume that the stock of raw materials is handled by the vendor. There is usually a time interval between ordering and delivering the raw materials, due to handling and transportation, among other factors.

A market is any external source having demand for endproducts. It is assumed that, if necessary, end-products are stocked at the show centers in the markets.

The supply chain consists of plants, where production, assembly and storage take place, that need to satisfy the demand of markets. It is assumed that each product is only manufactured/assembled in one plant, but plants can handle more than one product. Three types of resources are considered for the plants. In one type the extension of the base capacity can be performed at the first period and can be used along the whole time horizon. In another type it can be extended at the first period of any stage and can be used until the last period of the stage. And in the their type the base capacity can be extended independently for each period. The cost of capacity expansion for any resource is modeled as a piecewise discrete nondecreasing function.

The main TSCP decisions are those involving the raw material volume whose supplying is to be committed from vendors, the product volume to be processed and the stock volume of products to be stored in the plants, the component volume to be transported from the origin plants to the destination plants, the resources' capacity expansion, if any, and the end-product volume to be shipped from the plants to the markets. While the supplying commitment of raw material is done at the beginning of the planning horizon, the remaining decisions have to be taken in each period.

The notation used for the deterministic TSCP is as follows.

Sets

$\mathcal{T}$

$\mathcal{E}$

$\mathcal{J} E$

$\mathcal{J} S$,

$\mathcal{J}=\mathcal{J} E \cup \mathcal{J} S$

$\mathcal{D} S_{j}$, periods along the time horizon, lexicographically ordered, such that $t=1$ is the first one and $T=|\mathcal{T}|$ is the last period.

stages in the time horizon, lexicographically ordered, such that $\mathcal{T}^{e}$ is the set of consecutive periods in stage $e$, where $\mathcal{T}^{e} \cap \mathcal{T}^{e^{\prime}}=\emptyset$ for $e, e^{\prime} \in \mathcal{E}: e \neq e^{\prime}$. Note: $E=|\mathcal{E}|$ is the last stage. end-products.

subassemblies. products.

demand markets for end-product $j$, for $j \in$ $\mathcal{J} E$. Note: Without loss of generality, it is assumed that no subassembly has external demand. 
$\mathcal{D} S \quad=\bigcup_{j \in \mathcal{J} E} \mathcal{D} S_{j}$.

$\mathcal{I} R, \quad$ raw materials.

$\mathcal{I}, \quad$ components. Note: $\mathcal{I}=\mathcal{J} S \cup \mathcal{I} R$.

$\mathcal{I}_{j}, \quad \quad$ first tier components for product $j$, for $j \in \mathcal{J}$.

$R_{1}$, $\quad$ resources such that the capacity extension is performed at the first period of the time horizon, if any, and it can be used until the end of the horizon.

$R^{e}, \quad$ resources such that the capacity extension is performed at the first period of stage $e$, if any, and it can only be used until the last period of the stage, for $e \in \mathcal{E}$.

$R_{2}$, $\quad$ resources such that the capacity extension is performed at any period, if any, and it can only be used at that period.

$R=R_{1} \cup_{e \in \mathcal{E}} R^{e} \cup R_{2}$. Note: All the sets are disjoint.

$\mathcal{F}_{i}, \quad$ segments in the cost function for supplying commitment raw material $i$, for $i \in \mathcal{I} R$.

$\mathcal{H}_{r}, \quad$ segments for the capacity expansion of resource $r$, for $r \in R$.

\section{BoM parameters}

$c_{j}$, $\quad$ cycle time of product $j$, for $j \in \mathcal{J}$, and number of periods in the time interval from ordering raw material $j$ until it is ready for starting its delivering, for $j \in \mathcal{I} R$.

$N_{i, j}$, volume of first tier component $i$ that is required by the BoM to produce one unit of product $j$, for $i \in \mathcal{I}_{j}, j \in \mathcal{J}$.

\section{Time latency for availability}

$\tau_{i}, \quad$ number of time periods required to deliver component $i$ from its origin plant to its destination plant, for $i \in$ $\mathcal{J} S \cup \mathcal{I} R$.

$\tau_{d}$, delivery time, i.e., number of time periods to deliver endproduct $j$ to market $d$ after its completion, for $d \in \mathcal{D} S_{j}, j \in$ $\mathcal{J} E$.

\section{Production and product stock bounds}

$\underline{X}_{i, t}, \bar{X}_{i}, \quad$ minimum and maximum volume of raw material $i$ that can be ordered at time period $t$, respectively, if any, for $i \in \mathcal{I} R, t \in \mathcal{T}$, and minimum and maximum volume whose manufacturing/assembly can be released for product $i$ at time period $t$, respectively, if any, for $i \in \mathcal{J}, t \in \mathcal{T}$. Note: The upper bound $\bar{X}_{i}$ is stationary across the time horizon, but the assumption can be easily removed.

$\underline{S}_{j, t}, \bar{S}_{j}, \quad$ minimum and maximum volume of item $j$ that can be in stock at (the end of) time period $t$, respectively, for $j \in \mathcal{J} \bigcup \mathcal{I} R, t \in \mathcal{T}$.

$\underline{S e}_{d, t}, \overline{S e}_{d}$, minimum and maximum volume of end-product $j$ that can be in stock in (the show center of) market $d$ at (the end of) time period $t$, respectively, for $d \in \mathcal{D} S_{j}, j \in$ $\mathcal{J} E, t \in \mathcal{T}$.

\section{Resource coefficients}

$o_{r, j}$, amount of resource $r$ required to produce one unit of product $j$, for $r \in R, j \in \mathcal{J}$.

$O_{r}, \quad$ base capacity of resource $r$, for $r \in R_{1} \cup_{e \in \mathcal{E}} R^{e}$.

$O_{r}^{h}$, base capacity expansion of resource $r$ related to its $h$ th segment, for $h \in \mathcal{H}_{r}, r \in R_{1} \cup_{e \in \mathcal{E}} R^{e}$.

$O_{r, t}^{h}$, base capacity expansion of resource $r$ related to its $h$ th segment at time period $t$, for $h \in \mathcal{H}_{r}, r \in R_{2}, t \in \mathcal{T}$.

Cost coefficients

$\left(\overline{S C}_{i}^{f}, \bar{Y}_{i}^{f}\right)$, data defining the upper bound pair of the $f$ th segment of the supplying commitment cost function of raw material $i$, for $f \in \mathcal{F}_{i}, i \in \mathcal{I} R$, where $\overline{S C}_{i}^{f}$ is the cost, and
$\bar{Y}_{i}^{f}$ is the related volume. Note: Usually $\overline{S C}_{i}^{1}=0$ and $\bar{Y}_{i}^{1}=0$.

$f c_{j}$, fixed cost incurred when product $j$ is manufactured/assembled, for $j \in \mathcal{J}$, and fixed cost incurred when raw material $j$ is ordered, for $j \in \mathcal{I} R$.

$f c_{r}^{h}$, fixed cost associated with the $h$ th segment of the capacity expansion cost function of resource $r$, for $h \in$ $\mathcal{H}_{r}, r \in R_{1}$.

$f c_{r, t}^{h}$, fixed cost associated with the $h$ th segment of the capacity expansion cost function of resource $r$ at time period $t$, for $h \in \mathcal{H}_{r}, r \in R^{e}, e \in \mathcal{E}$, where $t$ is the first period in set $\mathcal{T}^{e}$.

$f c_{r, t}^{h}$, fixed cost associated with the $h$ th segment of the capacity expansion cost function of resource $r$ at time period $t$, for $h \in \mathcal{H}_{r}, r \in R_{2}, t \in \mathcal{T}$.

$h c_{j}, \quad$ unit holding cost of item $j$ at any time period, for $j \in$ $\mathcal{J} \cup \mathcal{I} R$.

hce $_{d}, \quad$ unit holding cost of end-product $j$ in market $d$ at any time period, for $d \in \mathcal{D} S_{j}, j \in \mathcal{J} E$.

$\ell c_{d, t}, \quad$ (resp. $b c_{d, t}$ ), unit lost demand (resp. unit backlogging) cost of end-product $j$ for market $d$ at time period $t$, for $d \in \mathcal{D} S_{j}, j \in \mathcal{J} E, t \in \mathcal{T}$.

$u_{i}$, unit reward of the surplus volume of raw material $i$ at the end of the time horizon, for $i \in \mathcal{I} R$.

In the next section, the STSCP modeling to study is presented as well as the challenges posed by the topology of the supply chain and the timing of the events in there.

\section{Uncertainty in tactical supply chain planning}

\subsection{Introduction}

The main uncertain parameters to consider in a realistic case along a given time horizon are production cost, available resource capacity, product demand and demand lost fraction. As usual, it is assumed that the realization of the uncertain parameters takes place at periods. One of the important decisions to be made in TSCP consists of determining the ordering time period for raw material supplying and subassembly readiness along the time horizon. There is usually a time interval between ordering and delivering the components (i.e., making them available) in the supply chain, due to handling of the ordering and transportation times that are required, among other factors. In the case that the time interval is not subject to specific constraints and costs related to the items' transportation and other factors, a deterministic model can consider that the ordering time is the same one as the delivering time. However, in the stochastic setting the related time interval is important, since the production and market environments can vary along the time interval between both events, see below. This is one of the challenges to be faced in this work.

Let scenario be one realization of the uncertain parameters along the periods of the time horizon, and a scenario group for a given period is the set of scenarios with the same realization of the uncertain parameters up to the period. This information structure can be visualized as the tree depicted in Fig.2, where each root-to-leaf path represents one specific scenario and corresponds to one realization of the whole set of the uncertain parameters. Each node in the tree can be associated with a scenario group in a one-to-one correspondence, such that two scenarios belong to the same group in a given period, provided that they have the same realization of the uncertain parameters up to the period. Let us point out that it is beyond the scope of this work to present a methodology for multi-period scenario tree generation and reduction; see e.g., Dupačová et al. (2000), Heitsch and Römisch (2009), 


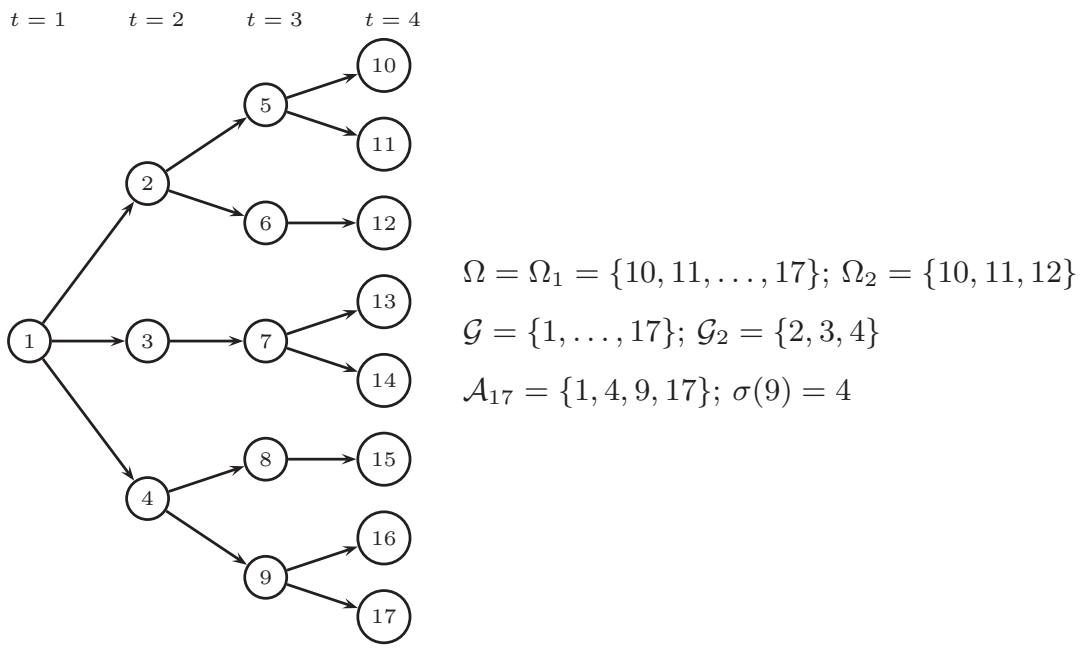

Fig. 2. Non-symmetric multi-period scenario tree.

Hoyland etal. (2003), Leövey and Römisch (2015) and Pflug and Pichler (2015a) for ways to perform both tasks.

Sets (lexicographically ordered in the scenario tree)

$\Omega, \quad$ scenarios.

$\mathcal{G}, \quad$ nodes in scenario tree.

$\mathcal{G}^{e} \subseteq \mathcal{G}, \quad$ nodes in stage $e$, for $e \in \mathcal{E}$.

$\mathcal{G}_{t} \subseteq \mathcal{G}$, nodes in period $t$, for $t \in \mathcal{T}$. Note: By construction, $\left|\mathcal{G}_{1}\right|=1$ and $1 \in \mathcal{G}_{1}$.

$\Omega_{g} \subseteq \Omega$, scenarios in group $g$, for $g \in \mathcal{G}$.

$\tilde{\mathcal{A}}_{g}, \quad$ node $g$ and its ancestors, for $g \in \mathcal{G}$. Notice that $\tilde{\mathcal{A}}_{1}$ is only included by node 1 and, then, $1 \in \mathcal{G}_{1}$.

$\mathcal{A}_{g} \subseteq \tilde{\mathcal{A}}_{g}$, nodes in $\tilde{\mathcal{A}}_{g}$ whose variables have nonzero elements in constraints related to node $g$ (including itself), for $g \in \mathcal{G}$.

$\mathcal{S}_{g}, \quad$ node $g$ and its successors, for $g \in \mathcal{G}$. Note: $\mathcal{S}_{g}=\{g\}$ for $g \in \mathcal{G}_{T}$.

$\mathcal{Q}^{e} \subseteq \mathcal{G}^{e}$, root nodes in the scenario subtrees starting in stage $e$, for $e \in \mathcal{E}$. As an illustration, let in Fig. 2 the set of stages $\mathcal{E}=\{1,2\}$ with $\mathcal{T}^{1}=\{1,2\}$ and $\mathcal{T}^{2}=\{3,4\}$, such that $\mathcal{G}^{1}=\{1,2,3,4\}$ and $\mathcal{G}^{2}=\{5, \cdots, 17\}$. So, e.g., $\mathcal{Q}^{2}=$ $\{5,6,7,8,9\}$, where node $q=7$ is the root node of the scenario subtree whose nodes are $\mathcal{S}^{2}=\{7,13,14\}$.

Other elements in the scenario tree

$w_{g}$, weight factor representing the likelihood that is associated with node $g$, for $g \in \mathcal{G}$. Note: $w_{g}=\sum_{\omega \in \Omega_{g}} w_{\omega}$, where $w_{\omega}$ gives the modeler-driven likelihood associated with scenario $\omega$ (where $w=g$ for $g \in \mathcal{G}_{T}$ such that it is a singleton set), for $\omega \in \Omega$, and $\sum_{g \in \mathcal{G}_{t}} w_{g}=1 \forall t \in \mathcal{T}$.

$\sigma(g)$, immediate ancestor node of node $g$, for $g \in \mathcal{G}$. By construction, $\sigma(1)=0$.

$t(g)$, period to which node $g$ belongs to, so, $g \in \mathcal{G}_{t(g)}$.

Uncertain parameters in node $g \in \mathcal{G}$

$D_{d}^{g}$, demand of end-product $j$ from market $d$ at time period $t(g)$, for $d \in \mathcal{D} S_{j}, j \in \mathcal{J} E$.

$f_{d}^{g}$, lost fraction of non-served accumulated demand of endproduct $j$ from market $d$, for $d \in \mathcal{D} S_{j}, j \in \mathcal{J} E$.

$O_{r}^{g}$, base capacity of resource $r$, for $r \in R_{2}$.

$p c_{j}^{g}$, unit production cost for product $j$, for $j \in \mathcal{J}$.

The so-called risk neutral measure in STSCP can be represented by the multi-period stochastic mixed $0-1$ model (1)-(16) that is presented next.

\subsection{Decision variables}

Continuous variables for raw material $i \in \mathcal{I} R . \quad y_{i}, \quad$ volume of raw material $i$ that is committed at the beginning of the time horizon.

$s c_{i}, \quad$ cost of supplying committed volume $y_{i}$ of raw material $i$.

$x_{j}^{g}$, volume of raw material $j$ that is ordered at (the beginning of) time period $t(g)$, for $j \in \mathcal{I} R$.

$z_{i}^{\omega}$, surplus volume of raw material $i$ at the end of the time horizon, for $\omega \in \Omega$.

$\beta_{i}^{f}, \quad[0,1]$ value defining the fraction used of the $f$ th segment of the piecewise linear, concave and nondecreasing supplying commitment cost function of raw material $i$. It belongs to the S2 sets for modeling that type of function. An S2 set Beale and Forrest (1976) is an ordered set of nonnegative variables $\left\{\beta_{i}^{f} \forall f \in \mathcal{F}_{i}\right\}$, such that the sum of the variables in the set must be 1 and no more than two members may be nonzero with the further condition that if there are as many as two they must be adjacent.

Continuous variables in node $\mathrm{g} \in \mathcal{G}$

$x_{j}^{g}$, released volume of product $j$ in the manufacturing/assembly line at (the beginning of) time period $t(g)$, for $j \in \mathcal{J}$.

$y_{d}^{g}$, shipped volume of end-product $j$ to market $d$ at (the end of) time period $t(g)$, for $d \in \mathcal{D} S_{j}, j \in \mathcal{J} E$.

$s_{j}^{g}, \quad$ stock volume of item $j$ at (the end of) time period $t(g)$,

for $j \in \mathcal{J} \cup \mathcal{I} R$.

$s e_{d}^{g}, \quad$ stock volume of end-product $j$ in market $d$ at (the end of) time period $t(g)$, for $d \in \mathcal{D} S_{j}, j \in \mathcal{J} E$.

$\ell_{d}^{g}$, lost demand of end-product $j$ in market $d$ at time period $t(g)$, for $d \in \mathcal{D} S_{j}, j \in \mathcal{J} E$.

$b_{d}^{g}$, demand backlog of end-product $j$ in market $d$ at (the end of) time period $t(g)$, for $d \in \mathcal{D} S_{j}, j \in \mathcal{J} E$.

Note: It is assumed that $s_{j}^{0}, s e_{d}^{0}, b_{d}^{0}$ are input data for any $j, d$.

0-1 variables for resources' capacity expansion and product release

$\alpha_{r}^{h}, \quad$ its value is 1 if the $h$ th segment of the capacity expansion of resource $r$ is performed at (the beginning of) the first period of the time horizon and otherwise 0 , for $h \in \mathcal{H}_{r}, r \in R_{1}$. Note 1: It can be used until the last period of the time horizon. Note 2: By construction, $\alpha_{r}^{0}=1$.

$\alpha_{r, h}^{q}, \quad$ its value is 1 if the $h$ th segment of the capacity expansion of resource $r$ is performed at (the beginning of) period $t(q)$ of node $q$ in stage $e(q)$, for $h \in \mathcal{H}_{r}, r \in R^{e}, q \in \mathcal{Q}^{e}, e \in$ 
$\mathcal{E}$. Note 1: It can be used until the last period of the stage. Note 2: By construction, $\alpha_{r, 0}^{q}=1$.

$\alpha_{r, h}^{g}, \quad$ its value is 1 if the $h$ th segment of the capacity expansion of resource $r$ is to performed at (the beginning of) period $t(g)$ of node $g$ and otherwise 0 , for $h \in \mathcal{H}_{r}, r \in R_{2}, g \in \mathcal{G}$. Note 1: It can only be used at the same period. Note 2: By construction, $\alpha_{r, 0}^{g}=1$.

$\delta_{j}^{g}$, its value is 1 if product $j$ is released in the manufacturing/assembly line for $j \in \mathcal{J}$, and it is 1 if raw material $j$ is ordered to vendor for $j \in \mathcal{I} R$, both at (the beginning of) time period $t(g)$ of node $g$ and otherwise, 0 , for $g \in \mathcal{G}$.

\subsection{Deterministic equivalent model, DEM}

The mixed 0-1 DEM can be expressed as follows,

$$
\begin{aligned}
& \min \sum_{i \in \mathcal{I} R} s c_{i}+\sum_{r \in R_{1}} \sum_{h \in \mathcal{H}_{r}} f c_{r}^{h} \alpha_{r}^{h} \\
& +\sum_{e \in \mathcal{E}} \sum_{q \in \mathcal{Q}^{e}} \sum_{r \in R^{e}} \sum_{h \in \mathcal{H}_{r}} w_{q} f c_{r, t(q)}^{h} \alpha_{r, h}^{q}+\sum_{g \in \mathcal{G}} \sum_{r \in R_{2}} \sum_{h \in \mathcal{H}_{r}} w_{g} f c_{r, t(g)}^{h} \alpha_{r, h}^{g} \\
& +\sum_{g \in \mathcal{G}} w_{g}\left[\sum_{j \in \mathcal{J}} p c_{j}^{g} x_{j}^{g}+\sum_{j \in \mathcal{J} \cup \mathcal{I} R} h c_{j} s_{j}^{g}+\sum_{d \in \mathcal{D} S}\left(h c e_{d} s e_{d}^{g}+\ell c_{d, t(g)} \ell_{d}^{g}\right.\right. \\
& \left.\left.+b c_{d, t(g)} b_{d}^{g}\right)\right]+\sum_{g \in \mathcal{G}} w_{g} \sum_{j \in \mathcal{J} \cup \mathcal{I R}} f c_{j} \delta_{j}^{g}-\sum_{i \in \mathcal{I} R} \sum_{\omega \in \Omega} w_{\omega} u_{i} z_{i}^{\omega}
\end{aligned}
$$

subject to

$$
\begin{array}{ll}
s c_{i} \equiv \sum_{f \in \mathcal{F}_{i}} \overline{S C}_{i}^{f} \beta_{i}^{f} & \forall i \in \mathcal{I} R \\
y_{i} \equiv \sum_{f \in \mathcal{F}_{i}} \bar{Y}_{i}^{f} \beta_{i}^{f} & \forall i \in \mathcal{I} R
\end{array}
$$

$\left(\beta_{i}^{f} \forall f \in \mathcal{F}_{i}\right)$ is an S2 set for raw material $i \quad \forall i \in \mathcal{I} R$

$y_{i}=\sum_{g \in \mathcal{A}_{\omega}} x_{i}^{g}+z_{i}^{\omega}$

$\forall \omega \in \Omega, i \in \mathcal{I} R$.

$$
s_{j}^{\sigma(g)}+x_{j}^{q}=\sum_{d \in \mathcal{D} S_{j}} y_{d}^{g}+s_{j}^{g} \quad \forall j \in \mathcal{J} E, g \in \mathcal{G},
$$

where $q \in \mathcal{A}_{g}: t(q)=t(g)-c_{j}+1$

$$
\begin{aligned}
s_{i}^{\sigma(g)}+x_{i}^{q}= & \sum_{j \in \mathcal{J}: i \in \mathcal{I}_{j}} N_{i, j} x_{j}^{h}+s_{i}^{g} \quad \forall h \in \mathcal{S}_{g}: t(h)=t(g) \\
& +\tau_{i}, g \in \mathcal{G}, i \in \mathcal{I},
\end{aligned}
$$

where $q \in \mathcal{A}_{g}: t(q)=t(g)-c_{i}+1$

$\underline{S}_{j, t(g)} \leq S_{j}^{g} \leq \bar{S}_{j} \quad \forall j \in \mathcal{J} \cup \mathcal{I} R, g \in \mathcal{G}$

$\underline{X}_{j, t(g)} \delta_{j}^{g} \leq x_{j}^{g} \leq \bar{X}_{j} \delta_{j}^{g}, \quad \delta_{j}^{g} \in\{0,1\} \quad \forall j \in \mathcal{J} \cup \mathcal{I} R, g \in \mathcal{G}$

$\sum_{j \in \mathcal{J}} o_{r, j} x_{j}^{g} \leq O_{r}+\sum_{h \in \mathcal{H}_{r}} O_{r}^{h} \alpha_{r}^{h} \quad \forall r \in R_{1}, g \in \mathcal{G}$

$\alpha_{r}^{h} \leq \alpha_{r}^{h-1}$ and $\alpha_{r}^{h} \in\{0,1\} \quad \forall h \in \mathcal{H}_{r}, r \in R_{1}$

$\sum_{j \in \mathcal{J}} o_{r, j} x_{j}^{g} \leq O_{r}+\sum_{h \in \mathcal{H}_{r}} O_{r}^{h} \alpha_{r, h}^{q} \quad \forall r \in R^{e}, g \in \mathcal{S}_{q} \cap \mathcal{G}^{e}, q \in \mathcal{Q}^{e}, e \in \mathcal{E}$

$$
\alpha_{r, h}^{q} \leq \alpha_{r, h-1}^{q} \text { and } \alpha_{r, h}^{q} \in\{0,1\} \quad \forall h \in \mathcal{H}_{r}, r \in R^{e}, q \in \mathcal{Q}^{e}, e \in \mathcal{E}
$$

$\sum_{j \in \mathcal{J}} o_{r, j} x_{j}^{g} \leq O_{r}^{g}+\sum_{h \in \mathcal{H}_{r}} O_{r, t(g)}^{h} \alpha_{r, h}^{g} \quad \forall r \in R_{2}, g \in \mathcal{G}$

$\alpha_{r, h}^{g} \leq \alpha_{r, h-1}^{g}$ and $\alpha_{r, h}^{g} \in\{0,1\} \quad \forall h \in \mathcal{H}_{r}, r \in R_{2}, g \in \mathcal{G}$

$s e_{d}^{\sigma(g)}+y_{d}^{q}+\ell_{d}^{g}+b_{d}^{g}=b_{d}^{\sigma(g)}+D_{d}^{g}+s e_{d}^{g} \quad \forall d \in \mathcal{D} S, g \in \mathcal{G}$.

where $q \in \mathcal{A}_{g}: t(q)=t(g)-\tau e_{d}$

$$
\begin{aligned}
& \ell_{d}^{g} \equiv f_{d}^{g}\left(b_{d}^{\sigma(g)}+D_{d}^{g}-s e_{d}^{\sigma(g)}-y_{d}^{q}\right) \geq 0 \quad \forall d \in \mathcal{D} S, g \in \mathcal{G} \\
& b_{d}^{g} \equiv\left(1-f_{d}^{g}\right)\left(b_{d}^{\sigma(g)}+D_{d}^{g}-s e_{d}^{\sigma(g)}-y_{d}^{q}\right) \geq 0 \quad \forall d \in \mathcal{D} S, g \in \mathcal{G}
\end{aligned}
$$

$\underline{S e}_{d, t(g)} \leq s e_{d}^{g} \leq \overline{S e}_{d} \forall d \in \mathcal{D} S, g \in \mathcal{G}$,

where it is assumed that $x_{j}^{q}=0$ and $y_{d}^{q}=0$ for $t(q) \leq 0$, and $x_{j}^{h}=0$ for $t(h)>T$.

\section{Objective function}

It consists of minimizing the supplying commitment raw material cost, the expected cost of production and stock of the items, resource capacity expansion, product stock at the markets and the penalty due to lost demand and backlogging in the scenarios along the time horizon, and minus the rewarding of the raw material surplus volume at the end of the time horizon.

Raw material supplying commitment cost function based on S2 sets

The constraint system (2) is the S2 sets object modeling for the raw material supplying commitment cost function. In addition, it imposes that the total supplying volume of a given raw material along the time horizon, $\sum_{g \in \mathcal{A}_{\omega}} x_{i}^{g}$, for any scenario $\omega \in \Omega$, plus the surplus, $z_{i}^{\omega}$, is equal to the supplying volume, $y_{i}$, that is committed at the beginning of the time horizon.

\section{Balance equations of end-products and components}

Constraint (3) is the balance equation for end-product $j$ in node $g$, where its cycle time $c_{j}$ needs to be taken into account. Without loss of generality it is assumed that no discharges are made during the process. Constraint (4) is the balance equation for component $i$ in node $g$. Note that this involves the set of products (subassemblies and end-products) $\left\{j \in \mathcal{J}: i \in \mathcal{I}_{j}\right\}$ that have component $i$ in the first tier of their BoM. Note: The cycle time and the product release time are taken into account. Constraint (5) impose upper and lower bounds on stock levels.

Upper and lower bounds for raw material supplying and product release

The Constraint (6) force the conditional bounds.

\section{Resource consumption}

The constraint system (7), (9), (11) bounds the resources' consumption, allowing the extension of the capacity of the resources, whose costs are expressed by a piecewise discrete nondecreasing functions. Given the character of the capacity expansion cost functions, the variable upper bounding system given by (8), (10), (12) forces the lexicographic order for the segments's commitment in the resources. 
Balance equations of product demand, lost demand and backlogging

Constraint (13) balance the total demand of market $d$ in node $g$, where the amount shipped to the markets is taken into account as well as the inventory, backlogged demand, and the possibility of lost demand. Constraints (14) and (15) ensure that the lost and backlogged demand are well defined. Constraint (16) impose upper and lower bounds on stock levels in (the show centers of) the markets. Note that $y_{d}^{q}$ is shipped at time period $t(q)=t(g)-\tau_{d}$ to satisfy the demand of end-product $j$ from market $d$ in the nodes in set $\mathcal{S}_{q} \cap \mathcal{G}_{t(q)+\tau_{d}}$. Observe also that if the stock is not enough for serving the backlog and demand, then the shipment to the market will be used. If this is not still enough, then it is partially lost and partially backlogged to be served at the immediate successor scenario nodes.

\subsection{Synthesized risk neutral STSCP model}

The model (1)-(16) can be synthesized in the following risk neutral (RN) model, whose aim consists of minimizing the expected tactical supply chain planning cost alone,

$$
\begin{aligned}
z_{R N}=\min & \sum_{g \in \mathcal{G}} w_{g}\left(a_{g} x_{g}+b_{g} y_{g}\right) \\
{[2 m m] \text { s.t. } } & \sum_{q \in \mathcal{A}_{g}}\left(A_{q}^{g} x_{q}+B_{q}^{g} y_{q}\right)=h_{g} \quad \forall g \in \mathcal{G} \\
& x_{g} \in\{0,1\}^{n x(g)}, y_{g} \in \mathbb{R}^{n y(g)} \quad \forall g \in \mathcal{G},
\end{aligned}
$$

where, for each node $g$ such that $g \in \mathcal{G}, x_{g}$ and $y_{g}$ denote the $n x(g)$ and $n y(g)$-vectors of the $0-1$ and continuous variables, respectively, $a_{g}$ and $b_{g}$ are the related objective function vectors, $A_{q}^{g}$ and $B_{q}^{g}$ are the constraints matrices whose nonzero coefficients give the influence of the appropriate $0-1$ and continuous variables from ancestor node $q$ in node $g$, respectively, and $h_{g}$ is the rhs vector.

\section{Time-based stochastic dominance measures}

Given the high variability on the uncertain parameters of the STSCP model (1)-(16), the time-inconsistent and time-consistent versions of risk averse measure are presented. It consists of a stochastic dominance functional as a multi-period oriented mixture of the first- and second-order stochastic dominance (SD) risk averse measures considered in Section 1.

\subsection{Time-inconsistent TSD measure}

To perform the TSCP cost risk reduction, the multi-period stochastic dominance risk averse measure proposed in Escudero et al. (2016a) is considered. In the following, we will refer to it as TSD. For that purpose, the operational cost function value is controlled for a subset of periods, say, $\tilde{\mathcal{T}} \subseteq \mathcal{T}$, such that a set of costrelated profiles, say $\mathcal{P}_{t}$, is considered, for $t \in \tilde{\mathcal{T}}$. A profile $p \in \mathcal{P}_{t}$ is defined by a 3 -tuple $\left(c^{p}, \bar{e}^{p}, \bar{\theta}^{p}\right)$, where $c^{p}$ is the threshold on the TSCP cost up to any node in $\mathcal{G}_{t} ; \bar{e}^{p}$ is the upper bound of the expected TSCP cost excess over threshold $c^{p}$ that is allowed in those nodes; and $\bar{\theta}^{p}$ is the upper bound on the probability of failing to satisfy the threshold. Additionally, let $\bar{c}_{t}$ denote the maximum TSCP cost that is allowed for any of those nodes.

Let $e_{g}^{p}$ denote the non-negative variable that defines the difference (if it is positive) between the TSCP cost up to node $g$ and threshold $c^{p}$, and $\theta_{g}^{p}$ is a $0-1$ variable such that its value is 1 if the cost up to node $g$ is greater than the threshold and otherwise, 0 .
The TSD measure can be expressed

$$
\begin{array}{rlrl}
z_{T S D}=\min & \sum_{g \in \mathcal{G}} w_{g}\left(a_{g} x_{g}+b_{g} y_{g}\right) & \\
{[2 m m] \text { s.t. }} & \sum_{q \in \mathcal{A}_{g}}\left(A_{q}^{g} x_{q}+B_{q}^{g} y_{q}\right)=h_{g} & & \\
& \sum_{q \in \tilde{\mathcal{A}}_{g}}\left(a_{q} x_{q}+b_{q} y_{q}\right)-e_{g}^{p} \leq c^{p} & & \forall g \in \mathcal{G}_{t}, p \in \mathcal{P}_{t}, t \in \tilde{\mathcal{T}} \\
& 0 \leq e_{g}^{p} \leq\left(\bar{c}_{t}-c^{p}\right) \theta_{g}^{p}, \theta_{g}^{p} \in\{0,1\} & & \forall g \in \mathcal{G}_{t}, p \in \mathcal{P}_{t}, t \in \tilde{\mathcal{T}} \\
& \sum_{g \in \mathcal{G}_{t}} w_{g} e_{g}^{p} \leq \bar{e}^{p} & & \forall p \in \mathcal{P}_{t}, t \in \tilde{\mathcal{T}} \\
& \sum_{g \in \mathcal{G}_{t}} w_{g} \theta_{g}^{p} \leq \bar{\theta}^{p} & & \forall p \in \mathcal{P}_{t}, t \in \tilde{\mathcal{T}} \\
& x_{g} \in\{0,1\}^{n x(g)}, y_{g} \in \mathbb{R}^{n y(g)} & & \forall g \in \mathcal{G} .
\end{array}
$$

The concept of the expected cost excess of the objective value on satisfying a given threshold have its roots in the Integrated Chance Constraints concept introduced in Klein (1986), see also Klein and van der Vlerk (2006).

Following the rationale in Pflug (2000) for the CVaR measure, it can be shown that TSD is a coherent risk measure, according to the standards set in Artzner etal. (1999); 2007). In other words, TSD satisfies the properties: translation invariance, positive homogeneity, monotonicity and convexity. In addition, it can also be shown Homem-de Mello and Pagnoncelli (2016) that the time-consistency of TSD, as defined below, depends on the bounds $\bar{e}^{p}$ and $\bar{\theta}^{p}$. The tighter these bounds are, the lower the consistency probability of TSD. Thus, in general, it is a time-inconsistent measure.

\subsection{Time-consistent ECSD measure}

The TSD risk reduction has an interesting add-value, since it controls the period-based peak of the TSCP cost in given intermediate periods while minimizing the expected cost for the whole time horizon, such that the longer the time horizon, the more usefulness of that strategy. However, its time inconsistency is a drawback. Notice that the outlooks of the scenarios that do not belong to the groups with a one-to-one correspondence with some nodes in the scenario tree (and, then, they are not to occur in those situations) have influence on the solution of those groups. To prove the statement it can be observed in model (18) that the solution for node $g \in \mathcal{G}_{t^{\prime}}, t^{\prime} \in \mathcal{T}$ is affected by the satisfaction of the risk constraint system related to period $t$ over all of its nodes, for $t \in \tilde{\mathcal{T}}: t^{\prime} \geq t$.

In this section, the expected conditional stochastic dominance (ECSD) measure is introduced. Here again a subset of periods $\tilde{\mathcal{T}}$ is given. However, the set of profiles, denoted by $\mathcal{P}_{g}$, are associated with node $g$, where $t(g) \in \tilde{\mathcal{T}}$, instead of been associated with period $t(g)$. A profile $p \in \mathcal{P}_{g}, t(g) \in \tilde{\mathcal{T}}$ consists of the 3-tuple $\left(c^{p}, \bar{e}^{p}, \bar{\theta}^{p}\right.$ ), where now $c^{p}$ is the TSCP cost threshold to be satisfied by any scenario in group $\Omega_{\mathrm{g}}$ from period 1 up to the last one, $T ; \bar{e}^{p}$ is the upper bound of the expected cost excess over threshold $c^{p}$ that is allowed for that group of scenarios; and $\bar{\theta}^{p}$ is the upper bound on the probability of failing to satisfy the threshold. The profile set $\mathcal{P}_{g}$ could be different for the nodes $g$ in set $\mathcal{G}_{t}, t \in \tilde{\mathcal{T}}$. (It is suggested to use this device for problems where there are high differences in the objective function coefficients between the scenarios that belong to different groups in the same period, at least). Additionally, let $\bar{c}_{g}$ denote the maximum TSCP cost that is allowed for any of scenario in set $\Omega_{\mathrm{g}}$. Note that period $T$ cannot be in $\tilde{\mathcal{T}}$ and, for practical reasons, the cardinality of $\tilde{\mathcal{T}}$ should be small.

Let the continuous variable $e_{\omega}^{p}$ and the $0-1$ variable $\theta_{\omega}^{p}$ for ECSD be as for TSD. The ECSD measure can be expressed 


$$
\begin{array}{rlrl}
z_{\mathrm{ECSD}}=\min & \sum_{g \in \mathcal{G}} w_{g}\left(a_{g} x_{g}+b_{g} y_{g}\right) & \\
{[2 m m] \text { s.t. }} & \sum_{q \in \mathcal{A}_{g}}\left(A_{q}^{g} x_{q}+B_{q}^{g} y_{q}\right)=h_{g} & & \\
& \sum_{q \in \tilde{\mathcal{A}}_{\omega}}\left(a_{q} x_{q}+b_{q} y_{q}\right)-e_{\omega}^{p} \leq c^{p} & & \forall p \in \mathcal{P}_{g}, \omega \in \Omega_{g}, g \in \mathcal{G}_{t}, t \in \tilde{\mathcal{T}} \\
& 0 \leq e_{\omega}^{p} \leq\left(\bar{c}_{g}-c^{p}\right) \theta_{\omega}^{p}, \theta_{\omega}^{p} \in\{0,1\} & & \forall p \in \mathcal{P}_{g}, \omega \in \Omega_{g}, g \in \mathcal{G}_{t}, t \in \tilde{\mathcal{T}} \\
& \sum_{\omega \in \Omega_{g}} w_{\omega} e_{\omega}^{p} \leq \bar{e}^{p} & & \forall p \in \mathcal{P}_{g}, g \in \mathcal{G}_{t}, t \in \tilde{\mathcal{T}} \\
& \sum_{\omega \in \Omega_{g}} w_{\omega} \theta_{\omega}^{p} \leq \bar{\theta}^{p} & & \forall p \in \mathcal{P}_{g}, g \in \mathcal{G}_{t}, t \in \tilde{\mathcal{T}} \\
& x_{g} \in\{0,1\}^{n x(g)}, y_{g} \in \mathbb{R}^{n y(g)} & & \forall g \in \mathcal{G} .
\end{array}
$$

It is worth to point out that the TSCP cost risk reduction in ECSD model (19) refers to the cost of the scenarios in group $\Omega_{g}$ up to the end of the time horizon. However, the TSCP cost risk reduction in TSD model (18) refers to the cost of the same scenario group, but it is only up to node $g$, for $g \in \mathcal{G}_{t}, t \in \tilde{\mathcal{T}}$. Also note that if $\tilde{\mathcal{T}}$ is singleton and in both measures the cost risk reduction is desired to be performed for all scenarios at the end of the time horizon, then it happens that $z_{E C S D}=z_{T S D}$ for $\tilde{\mathcal{T}}=\{1\}$ in ECSD and $\tilde{\mathcal{T}}=\{T\}$ in TSD.

The concept of expected conditional risk measure (ECRM) was introduced in Homem-de Mello and Pagnoncelli (2016), where the time-consistency is defined and proved, see also Asamov and Ruszczynski (2015), Pflug and Pichler (2015b), Rudloff et al. (2014), Ruszczyński (2010) and Shapiro (2009). Notice that the proof only requires that the measure has the properties of translationinvariance and monotonicity. In what follows the time-consistency property of ECSD in (19) is proved to be a member of the family of ECRMs.

Let $\hat{x}_{q}$ and $\hat{y}_{q} \forall q \in \mathcal{G}$ denote the vectors with the values of the variables in the vectors $x_{q}$ and $y_{q}$ of model (19), for any of its optimal solutions. Let $\tilde{z}_{E C S D_{g}}$ denote the value of the sum of the terms in the objective function of model (19) related to the optimal values $\hat{x}_{q}$ and $\hat{y}_{q}$, for $q \in \tilde{\mathcal{A}}_{g} \cup \mathcal{S}_{g}$ for any node $g \in \mathcal{G}$, i.e., $\tilde{z}_{E C S D_{g}}=\sum_{q \in \tilde{\mathcal{A}}_{g} \cup \mathcal{S}_{g}} w_{q}\left(a_{q} \hat{x}_{q}+b_{q} \hat{y}_{q}\right)$.

Let us consider the submodel (20) of model (19) related to node $g$, for $g \in \mathcal{G}$, whose elements are as follows:

- The subtree from the original scenario tree that supports the submodel is given by the node set $\tilde{\mathcal{A}}_{g} \cup \mathcal{S}_{g}$, i.e., the nodes up to node $g$ plus the subtree rooted in $g$ up to the leaf nodes.

- The input data of the submodel is taken from the appropriate nodes in model (19).

- The variables in the vectors $x_{g}$ and $y_{q} \forall q \in \mathcal{A}_{g} \backslash\{g\}$ are fixed in submodel (20) to the related values in the vectors $\hat{x}_{q}$ and $\hat{y}_{q}$ in the optimal solution of model (19) that is being considered.

$$
\begin{aligned}
& z_{E C S D_{g}}=\min \sum_{q \in \tilde{\mathcal{A}}_{g} \cup \mathcal{S}_{g}} w_{q}\left(a_{q} x_{q}+b_{q} y_{q}\right) \\
& \text { s.t. } \sum_{q \in \mathcal{A}_{g^{\prime}}}\left(A_{q}^{g^{\prime}} x_{q}+B_{q}^{g^{\prime}} y_{q}\right)=h_{g^{\prime}} \\
& \sum_{q \in \tilde{\mathcal{A}}_{\omega}}\left(a_{q} x_{q}+b_{q} y_{q}\right)-e_{\omega}^{p} \leq c^{p} \\
& 0 \leq e_{\omega}^{p} \leq\left(\bar{c}_{g}-c^{p}\right) \theta_{\omega}^{p}, \theta_{\omega}^{p} \in\{0,1\} \\
& \sum_{\omega \in \Omega_{g^{\prime}}} w_{\omega} e_{\omega}^{p} \leq \bar{e}^{p} \\
& \sum_{\omega \in \Omega_{g^{\prime}}} w_{\omega} \theta_{\omega}^{p} \leq \bar{\theta}^{p} \\
& x_{q}=\hat{x}_{q}, y_{q}=\hat{y}_{q} \\
& x_{q} \in\{0,1\}^{n x(q)}, y_{q} \in \mathbb{R}^{n y(q)} \\
& \forall g^{\prime} \in \mathcal{S}_{g} \\
& \forall p \in \mathcal{P}_{g^{\prime}}, \omega \in \Omega_{g^{\prime}}, g^{\prime} \in \mathcal{G}_{t}, t \in \tilde{\mathcal{T}}: t \geq t(g) \\
& \forall p \in \mathcal{P}_{g^{\prime}}, \omega \in \Omega_{g^{\prime}}, g^{\prime} \in \mathcal{G}_{t}, t \in \tilde{\mathcal{T}}: t \geq t(g) \\
& \forall p \in \mathcal{P}_{g^{\prime}}, g^{\prime} \in \mathcal{G}_{t}, t \in \tilde{\mathcal{T}}: t \geq t(g) \\
& \forall p \in \mathcal{P}_{g^{\prime}}, g^{\prime} \in \mathcal{G}_{t}, t \in \tilde{\mathcal{T}}: t \geq t(g) \\
& \forall q \in \tilde{\mathcal{A}}_{g} \backslash\{g\} \\
& \forall q \in \tilde{\mathcal{A}}_{g} \cup \mathcal{S}_{g}
\end{aligned}
$$

It is easy to show that the ECSD measure is time-consistent, i.e., $\tilde{z}_{E C S D_{g}}=z_{E C S D_{g}}$, for any node $g \in \mathcal{G}$, any scenario tree, any pro- file set, any period subset, and any other input data. Let us assume that the decisions in a given problem have been taken up to node $\sigma(g)$ according to the solution obtained in the original model (19) solved at period $t=1$. Then, the rationale behind a time-consistent risk averse measure is that the solution value to be obtained for node set $\mathcal{S}_{g}$ (where remember node $g$ is also included) in the scenario tree for the related submodel (20) solved at period $t(g)$ should have the same value as in the original model solved at period $t=1$.

Notice that $\Omega_{g^{\prime}} \subset \Omega_{g}$ for $t\left(g^{\prime}\right)>t(g)$. And, given the structure of ECSD for risk reduction, it results that ECSD, as any other ECRM with cross-node constraints, is very appropriate for considering the stochastic nested decomposition (SND) methodology as a suitable tool for solving large sized instances. See also below and Cristobal et al. (2009), Escudero etal. (2013), Escudero etal. (2015) and Zou etal. (2016), among others. Note: The SND variant where there is not any Markovian relationship between the uncertainty in successive periods was introduced in Pereira and Pinto (1985).

\section{Markovian-based stochastic dynamic programming}

In this section the matheuristic presented in Escudero etal. (2015) is extended to deal with the cross-scenario constraints in ECSD model (19). The new algorithm, so-called SDP-ECSD, considers the set of stages $\mathcal{E}$, so that the stochastic problem is decomposed into a collection of inter-connected subproblems. A key item in the proposed matheuristic algorithm is the concept of the socalled Expected Future Value (EFV) curves. These curves estimate the impact of the linking decisions made at a given stage on the objective function value related to the future stages. SDP-ECSD is a SND variant where the linking variables between consecutive periods can also be continuous variables besides the $0-1$ variables.

The new proposal is designed to deal with the ECSD model (19) and it is not a trivial task. Apart from the problem size that is allowed, another major challenge is to deal with the (probably, numerous) cross-scenario constraints that link the scenarios in set $\Omega_{g}$, for $g \in \mathcal{G}_{t}, t \in \tilde{\mathcal{T}}$. To deal with those constraints, a mechanism is devised that distributes the TSCP cost risk reduction bounds $\bar{e}^{p}$ and $\bar{\theta} p$, for $p \in \mathcal{P}_{g}$, among the immediate successors of any given subproblem.

\subsection{The subproblems}

In this section the subproblems and the EFV curve concept and purpose are presented. Consider the additional notation:

$\mathcal{R}^{e} \subseteq \mathcal{G}^{e}, \quad$ set of root nodes to the subtrees of stage $e$. Note: Without loss of generality, let us assume that the node set $\{g \in \mathcal{G}: t(g) \in \tilde{\mathcal{T}}\}$ where to perform the TSCP cost risk reduction is a subset of $\bigcup_{e \in \mathcal{E}} \mathcal{R}^{e}$, i.e., only those root nodes can be a subject of TSCP cost risk reduction.

$\mathcal{C}_{r} \subseteq \mathcal{G}^{e}, \quad$ set of nodes in $\mathcal{G}^{e}$ that belong to the subtree rooted in node $r$, for $r \in \mathcal{R}^{e}, e \in \mathcal{E}$.

$\mathcal{L}_{r} \subseteq \mathcal{C}_{r}, \quad$ set of leaf nodes in $\mathcal{C}_{r}$, for $r \in \mathcal{R}^{e}, e \in \mathcal{E}$.

$\mathcal{S}_{g}^{1} \subseteq \mathcal{S}_{g} \backslash\{g\}$, set of immediate successors of node $g$, for $g \in \mathcal{G}$ : $t(g)<T$ (i.e., $q \in \mathcal{S}_{g}^{1}$ provided that $\left.t(q)=t(g)+1\right)$.

$\tilde{\mathcal{A}}_{\ell} \subseteq \mathcal{A}_{\ell}, \quad$ set consisting of leaf node $\ell \in \mathcal{L}_{r}$ and its ancestors, such that their variables have nonzero elements in the constraints associated with the nodes in the immediate successor subproblems to node $\ell$. Observe that those nodes are in set $\bigcup_{r^{\prime} \in \mathcal{S}_{\ell}^{1}} \mathcal{C}_{r^{\prime}}$. On the other hand, the nodes $r^{\prime}$ are the root ones in set $\mathcal{S}_{\ell}^{1}$ (i.e., the root nodes in the related supporting subtrees), for $\ell \in \mathcal{L}_{r}, r \in \mathcal{R}^{e}, e \in \mathcal{E} \backslash\{E\}$. 


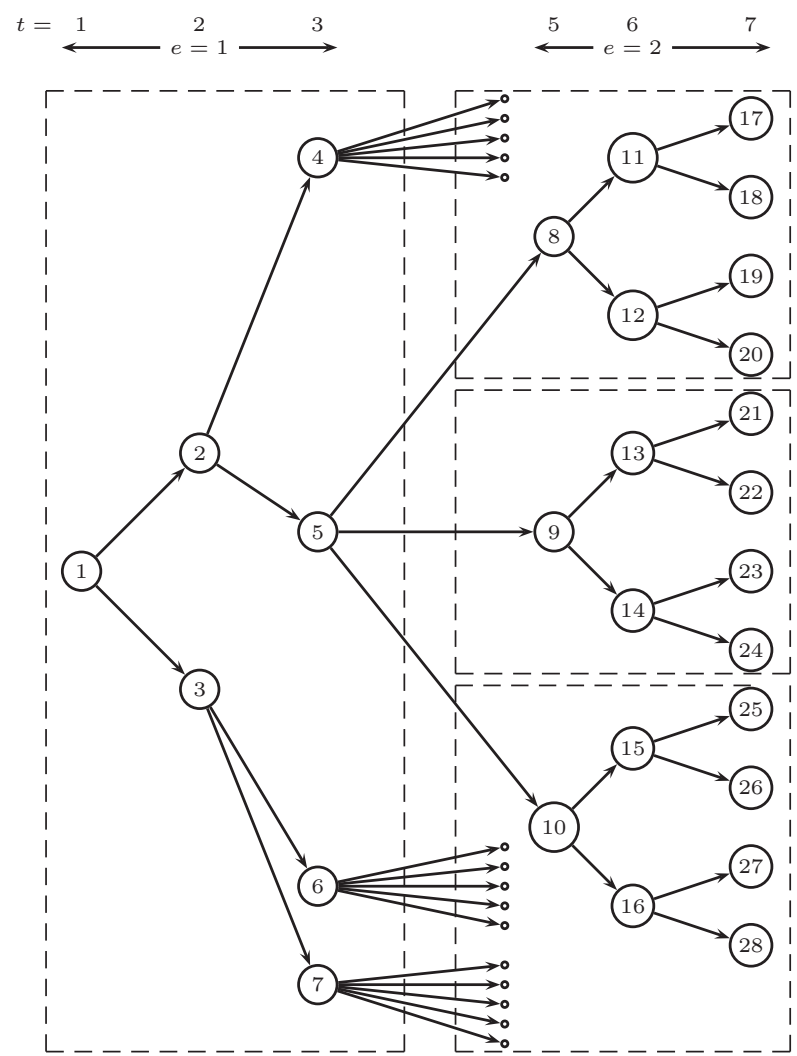

$$
\begin{aligned}
& \mathcal{G}^{1}=\{1,2, \ldots, 7\} \\
& \mathcal{R}^{2}=\{8,9,10\} \\
& \mathcal{C}_{8}=\{8,11,12,17,18,19,20\} \\
& \mathcal{L}_{1}=\{4,5,6,7\}
\end{aligned}
$$

Fig. 3. Breaking the time horizon into stages.

$\mathcal{P}^{r}$, set of profiles $\left\{p \in \mathcal{P}_{g}, g \in \tilde{\mathcal{A}}_{r}: t(g) \in \tilde{\mathcal{T}}\right\}$, for $r \in$ $\mathcal{R}^{e}, e \in \mathcal{E}$.

Fig. 3 illustrates the sets above where the time horizon is split into two stages.

Consider $r$ for $r \in \mathcal{R}^{e}, e \in \mathcal{E}$. Let $\hat{x}_{q}$ and $\hat{y}_{q}$ be given values of the vectors of variables $x_{q}$ and $y_{q}$, respectively, for $q \in \tilde{\mathcal{A}}_{\sigma(r)}$. There are two types of variables for the TSCP cost risk reduction functional in the $r$-rooted model (21)-(33). The first type is included by the continuous variable $e_{r^{\prime}}^{p}$ and the $0-1$ variable $\theta_{r^{\prime}}^{p}$, for $p \in$ $\mathcal{P}^{r}, r^{\prime} \in \mathcal{S}_{\ell}^{1}, \ell \in \mathcal{L}_{r}$, where $e<E$, that result from the distribution of the ECSD bounds $\hat{e}_{r}^{p}$ and $\hat{\theta}_{r}^{p}$, respectively, among the immediate successor subprograms rooted in the nodes $r^{\prime}$. (Notice that the models rooted in the nodes in set $\mathcal{R}^{E}$ do not need to perform that distribution). The second type of variables is included by the continuous variable $e^{r^{\prime} p}$ and the $0-1$ variable $\theta^{r^{\prime} p}$, such that $e^{r^{\prime} p}$ gives the estimation of the TSCP cost excess of the expected scenario in group $\Omega_{r^{\prime}}$ over threshold $c^{p}$, and $\theta^{r^{\prime} p}$ is the related $0-$ 1 variable (such that $\theta^{r^{\prime} p}=1$ for $e^{r^{\prime} p}>0$ and otherwise, 0 ), for $p \in \mathcal{P}^{r}, r^{\prime} \in \mathcal{S}_{\ell}^{1}, \ell \in \mathcal{L}_{r}$. Notice that the latter type of variables is aimed to help to satisfying the ECSD bounds. Note: The satisfaction of those bounds is increasingly important though the stages until reaching the last one. The ECSD bounds for $p \in \mathcal{P}_{r}: t(r) \in \tilde{\mathcal{T}}$ are as follows: $\hat{e}_{r}^{p}=\bar{e}^{p}$ (original bound) and $\hat{\theta}_{r}^{p}=\bar{\theta}^{p}$ (original bound). On the other hand, those ECSD bounds $\hat{e}_{r}^{p}$ and $\hat{\theta}_{r}^{p}$, for $p \in \mathcal{P}^{r} \backslash \mathcal{P}_{r}$, if any, are the values of the variables $e_{r}^{p}$ and $\theta_{r}^{p}$, respectively. Those values are taken from the solution of the $r^{\prime \prime}$-rooted model (21)(33), for $r^{\prime \prime} \in \mathcal{R}^{e-1}, e \in \mathcal{E} \backslash\{1\}$, such that $r \in \mathcal{S}_{\ell^{\prime \prime}}^{1}, \ell^{\prime \prime} \in \mathcal{L}_{r^{\prime \prime}}$.

Let $\lambda_{r^{\prime}}^{\prime}(\cdot)$ be a function that gives an approximation of the future TSCP cost in the set of scenarios $\Omega_{r^{\prime}}$ for $r^{\prime} \in \mathcal{S}_{\ell}^{1}, \ell \in \mathcal{L}_{r}, r \in$ $\mathcal{R}^{e}, e \in \mathcal{E} \backslash E$, related to the set $\left\{e^{\prime} \in \mathcal{E}: e^{\prime}>e\right\}$. This function has the argument $()=.\left(x_{q}, y_{q} \forall q \in \tilde{\tilde{\mathcal{A}}}_{\sigma(r)} ; e_{r}^{p}, \theta_{r}^{p} \forall p \in \mathcal{P}^{r}\right)$.

$$
\begin{aligned}
F_{r}^{\prime}(.) & =\min \sum_{\ell \in \mathcal{L}_{r}} w_{\ell}\left[\sum_{q \in \tilde{\mathcal{A}}_{\ell}}\left(a_{q} x_{q}+b_{q} y_{q}\right)+\sum_{r^{\prime} \in \mathcal{S}_{\ell}^{1}} \frac{w_{r^{\prime}}}{w_{\ell}} \lambda_{r^{\prime}}^{\prime}(\cdot)\right] \\
& +\sum_{p \in \mathcal{P}^{r}}\left(M_{r}^{p^{1}} \epsilon_{r}^{p^{1}}+M_{r}^{p^{2}} \epsilon_{r}^{p^{2}}\right)
\end{aligned}
$$

subject to

$$
\begin{aligned}
& \sum_{q \in \mathcal{A}_{g}}\left(A_{q}^{g} x_{q}+B_{q}^{g} y_{q}\right)=h_{g} \quad \forall g \in \mathcal{C}_{r} \\
& \sum_{q \in \mathcal{A}_{\ell}}\left(a_{q} x_{q}+b_{q} y_{q}\right)+\frac{1}{\left|\Omega_{r^{\prime}}\right|} \lambda_{r^{\prime}}^{\prime}(\cdot)-e^{r^{\prime} p} \leq c^{p} \\
& \forall p \in \mathcal{P}^{r}, r^{\prime} \in \mathcal{S}_{\ell}^{1}, \ell \in \mathcal{L}_{r} \\
& 0 \leq e^{r^{\prime} p} \leq\left(\bar{c}-c^{p}\right) \theta^{r^{\prime} p}, \quad \theta^{r^{\prime} p} \in\{0,1\} \quad \forall p \in \mathcal{P}^{r}, r^{\prime} \in \mathcal{S}_{\ell}^{1}, \ell \in \mathcal{L}_{r}
\end{aligned}
$$

$\sum_{\ell \in \mathcal{L}_{r}} \sum_{r^{\prime} \in \mathcal{S}_{\ell}^{1}} w_{r^{\prime}} e^{r^{\prime} p} \leq e_{r}^{p}+\epsilon_{r}^{p^{1}} \quad \forall p \in \mathcal{P}^{r}$

$\sum_{\ell \in \mathcal{L}_{r}} \sum_{r^{\prime} \in \mathcal{S}_{\ell}^{1}} w_{r^{\prime}} \theta^{r^{\prime} p} \leq \theta_{r}^{p}+\epsilon_{r}^{p^{2}} \quad \forall p \in \mathcal{P}^{r}$

$\sum_{\ell \in \mathcal{L}_{r}} \sum_{r^{\prime} \in \mathcal{S}_{\ell}^{1}} e_{r^{\prime}}^{p}=e_{r}^{p} \quad \forall p \in \mathcal{P}^{r}: e<E$

$\sum_{\ell \in \mathcal{L}_{r}} \sum_{r^{\prime} \in \mathcal{S}_{\ell}^{1}} \theta_{r^{\prime}}^{p}=\theta_{r}^{p} \quad \forall p \in \mathcal{P}^{r}: e<E$ 
$x_{q}=\hat{x}_{q}, y_{q}=\hat{y}_{q} \quad \forall q \in \tilde{\mathcal{A}}_{\sigma(r)}: t(r)>1$

$e_{r}^{p}=\hat{e}_{r}^{p}, \theta_{r}^{p}=\hat{\theta}_{r}^{p} \quad \forall p \in \mathcal{P}^{r}$

$x_{q} \in\{0,1\}^{n x(q)}, y_{q} \in \mathbb{R}^{n y(q)} \quad \forall q \in \tilde{\mathcal{A}}_{\ell}, \ell \in \mathcal{L}_{r}$

$$
\begin{aligned}
& e^{r^{\prime} p} \in \mathbb{R}^{+}, \theta^{r^{\prime} p} \in\{0,1\}, e_{r}^{p} \in \mathbb{R}^{+}, \theta_{r}^{p} \in \mathbb{R}^{+} \\
& \forall p \in \mathcal{P}^{r}, r^{\prime} \in \mathcal{S}_{\ell}^{1}, \ell \in \mathcal{L}_{r}
\end{aligned}
$$

$$
\epsilon_{r}^{p^{1}} \in \mathbb{R}^{+}, \epsilon_{r}^{p^{2}} \in \mathbb{R}^{+} \quad \forall p \in \mathcal{P}^{r} .
$$

Before describing the ingredients of the above formulation, let us highlight some important differences between this subtree supported subproblem and the ECSD model (19) supported by the whole tree. First, the formulation has fixed the $x, y$-variables of ancestor nodes to the subtree rooted in node $r$, see constraints (29). Second, with the aim that the objective function value of subproblem (21)-(33) be a good approximation of the objective function value of scenario set $\Omega_{r}$ in problem (19), the expected value of the terms related to the successor subproblems is represented by the functions $\lambda_{r^{\prime}}^{\prime}(\cdot)$ for the sets of scenarios $\Omega_{r^{\prime}}$, for $r^{\prime} \in \mathcal{S}_{\ell}^{1}, \ell \in \mathcal{L}_{r}$. Notice that those sets are the scenario groups with a one-to-one correspondence with the immediate successor nodes to the leaf ones in the subtree rooted in node $r$. So, those functions are added to objective function (21). Therefore, in the constraints (23), $\lambda_{r^{\prime}}^{\prime}(\cdot)$ is also divided by the number of scenarios in set $\Omega_{r^{\prime}}$. Note: It is assumed that $\frac{1}{\left|\Omega_{r^{\prime}}\right|} \lambda_{r^{\prime}}^{\prime}(\cdot)$ represents an approximation to the expected cost of any scenario in that group. Observe that no decisions are taken prior to the root node $r=1$, while no estimation is required in the subproblems in the last stage $E$, since the time horizon ends there. So, any reference to $\mathcal{A}_{1} \backslash\{1\}$ or $\lambda_{\ell}^{\prime}(\cdot)$, for $\ell \in \mathcal{G}_{T}$ should not be considered in model (21)-(33).

The terms in the objective function (21) related to leaf node $\ell \in \mathcal{L}_{r}$ give the expected TSCP cost along the time horizon for the set of scenarios in $\Omega_{\ell}$. The first term is the cost related to the ancestor node set $\tilde{A}_{\ell}$ of node $\ell$ (including itself), and the second term is an approximation of the expected cost of its successor subproblems (whose nodes are in set $\mathcal{S}_{\ell}$ ). The last term in the objective function is the penalization of the infeasibility of the ECSD constraints (25) and (26).

Constraint (22) is related to the nodes in the subproblem. Each constraint (23) defines the variable $e^{r^{\prime} p}$ that takes the expected excess of the TSCP cost over threshold $c^{p}$ for any scenario in group $\Omega_{r^{\prime}}, r^{\prime} \in \mathcal{S}_{\ell}^{1}, \ell \in \mathcal{L}_{r}$. Notice that the expected excess is an approximation. Constraint (24) force the variable $\theta^{r^{\prime} p}=1$ for $e^{r^{\prime} p}=1>0$ and otherwise, 0 .

Constraints (25) and (26) are the counterparts of the ECSD constraints in model (19), where the new (slack) variables $\epsilon_{r}^{p^{1}}$ and $\epsilon_{r}^{p^{2}}$ ensure those constraints' feasibility, respectively. The variables are penalized in the objective function by using the big $M$ type parameters $M_{r}^{p^{1}}$ and $M_{r}^{p^{2}}$, for the $p$ th profile with the aim of having them equal to zero at the end of the algorithm's execution. Note: The value of those parameters is a non-decreasing function of the SDP-ECSD iteration number, say ite. Observe that the left hand side of constraint (25) gives the (approximate) expected cost excess for the whole set of scenarios $\Omega_{r}$.

Constraints (27) (resp. (28)) distribute the ECSD variable $e_{r}^{p}$ (resp. $\theta_{r}^{p}$ ) among the subproblems whose root nodes $r^{\prime}$ are the immediate successors of the leaf nodes $\ell$, for $\ell \in \mathcal{L}_{r}$. The distribution is made at random for the SDP-ECSD first iteration where a solution to the original problem is obtained in its forward scheme.
However, when the iterations go on, (hopefully) function $\lambda_{r^{\prime}}^{\prime}(\cdot)$ will correctly assign the $e$ - and $\theta$-variables to the successor subproblems.

Constraints (29) (resp. (30)) fix the variables in vectors $x_{q}$ and $y_{q}$ (resp. variables $e_{r}^{p}$ and $\theta_{r}^{p}$ ).

\subsection{The EFV curves}

Notice that, in general, $\lambda_{r^{\prime}}^{\prime}(\cdot)$, for $r^{\prime} \in \mathcal{S}_{\ell}^{1}, \ell \in \mathcal{L}_{r}, r \in \mathcal{R}^{e}, e \in \mathcal{E} \backslash$ $\{E\}$ is difficult to compute. The matheuristic SDP-ECSD approach approximates it by a piecewise linear convex function, whose related variable is notated as $\lambda_{r^{\prime}}$; it will be referred to as the expected future value (EFV). The matheuristic is an iterative algorithm where each iteration consists of a forward scheme followed by a backward scheme. The forward scheme is intended to improve the current solution, where the single subproblem in the first stage is solved and the linking variables are fed to subproblems in the second stage, which in turn are solved. This process is repeated for each stage until the last one is reached, yielding a new solution. The backward scheme refines the current EFV curves by using all already obtained solutions. To refine those curves, strong duality theory is applied by starting with the subproblems supported for the subtrees rooted in the nodes $r^{\prime}$ for $r^{\prime} \in \mathcal{R}^{E}$ around the set of solutions obtained so far up to stage $E$ (in the related forward scheme up to the current iteration ite). As a result, valid cuts are obtained for approximating function $\lambda_{r^{\prime}}^{\prime}(\cdot)$. So, its updated constraint system is appended to the immediate ancestor subproblem. It is supported by the subtree rooted in node $r$, for $r \in \mathcal{R}^{E-1}$, such that $r^{\prime} \in \mathcal{S}_{\ell}^{1}$ for $\ell \in \mathcal{L}_{r}$. The process is repeated until the second stage is reached, such that its cut-based updated constraint systems are appended to the subproblem related to stage $e=1$. It is worth to point that, by construction, each subproblem to solve at any stage (but the last one) has not full information about the value of the objective function terms related to later stages, but an estimation. Notice that the later the stage and the deeper the iteration are in the algorithm's execution, the more precise the information is.

So, let $\hat{X}_{\ell}^{i}, \ell \in \mathcal{L}_{r}$ denote the solution vector obtained while solving the subproblem (36)-(40) supported by the subtree rooted in node $r \in \mathcal{R}^{e}$ for $e \in \mathcal{E} \backslash\{E\}$ in the forward scheme at iteration $i$, for $i=1, \cdots$, ite. It can be expressed as follows,

$\hat{X}_{\ell}^{i} \equiv\left(\hat{x}_{q}^{i}, \hat{y}_{q}^{i} \forall q \in \tilde{\tilde{\mathcal{A}}}_{\ell} ; \hat{e}_{r^{\prime}}^{p^{i}}, \hat{\theta}_{r^{\prime}}^{p^{i}} \forall p \in \mathcal{P}^{r}, r^{\prime} \in \mathcal{S}_{\ell}^{1}\right)$.

Additionally, let $\mathcal{Z}_{\mathrm{r}^{\prime}}$ denote the set of so-called reference levels, for $r^{\prime} \in \mathcal{S}_{\ell}^{1}, \ell \in \mathcal{L}_{r}, r \in \mathcal{R}^{e}, e \in \mathcal{E} \backslash\{E\}$, where the $z$ th one is included by vector

$\left(\left(\pi_{q}^{z}\right)_{r^{\prime}},\left(\gamma_{q}^{z}\right)_{r^{\prime}} \forall q \in \tilde{\tilde{\mathcal{A}}}_{\ell} ;\left(\delta^{p^{z}}\right)_{r^{\prime}},\left(\tau^{p^{z}}\right)_{r^{\prime}} \forall p \in \mathcal{P}^{r},\left(\mu^{z}\right)_{r^{\prime}}\right)$,

where $\left(\pi_{q}^{z}\right)_{r^{\prime}},\left(\gamma_{q}^{z}\right)_{r^{\prime}}$ and $\left(\delta^{p^{z}}\right)_{r^{\prime}},\left(\tau^{p^{z}}\right)_{r^{\prime}}$ are the dual vectors of the constraints (29) and (30), respectively, when solving the subproblem $F_{r^{\prime}}\left(\hat{X}_{\sigma\left(r^{\prime}\right)}^{i}\right)(36)-(40)$, and $\left(\mu^{z}\right)_{r^{\prime}}(45)$ is the constant of the cut for the $z$ th reference level in set $\mathcal{Z}_{r^{\prime}}$ to define the EFV curve approximation in the related immediate ancestor $r$-rooted subproblem.

Consider again $r \in \mathcal{R}^{e}, e \in \mathcal{E} \backslash\{E\}$. Let the variable $\lambda_{r^{\prime}}$ denote the approximated value of the EFV curve $\lambda_{r^{\prime}}^{\prime}(\cdot)$. So, the subproblem (21)-(33) that defines the objective function $F_{r}^{\prime}(\cdot)$, at iteration $i$, can be approximated as follows,

$$
\begin{aligned}
F_{r}\left(\hat{X}_{\sigma(r)}^{i}\right) & =\min \sum_{\ell \in \mathcal{L}_{r}} w_{\ell}\left[\sum_{q \in \tilde{\mathcal{A}}_{\ell}}\left(a_{q} x_{q}+b_{q} y_{q}\right)+\sum_{r^{\prime} \in \mathcal{S}_{\ell}^{1}} \frac{w_{r^{\prime}}}{w_{\ell}} \lambda_{r^{\prime}}\right] \\
& +\sum_{p \in \mathcal{P}^{r}}\left(M_{r}^{p^{1}} \epsilon_{r}^{p^{1}}+M_{r}^{p^{2}} \epsilon_{r}^{p^{2}}\right)
\end{aligned}
$$

subject to

Constraints (21)-(22) and (24)-(33) 


$$
\sum_{q \in \tilde{\mathcal{A}}_{\ell}}\left(a_{q} x_{q}+b_{q} y_{q}\right)+\frac{1}{\left|\Omega_{r^{\prime}}\right|} \lambda_{r^{\prime}}-e^{r^{\prime} p} \leq c^{p} \quad \forall p \in \mathcal{P}^{r}, \quad r^{\prime} \in \mathcal{S}_{\ell}^{1}, \ell \in \mathcal{L}_{r}
$$

$$
\begin{aligned}
\lambda_{r^{\prime}} & \geq\left(\mu^{z}\right)_{r^{\prime}}+\sum_{q \in \tilde{\tilde{A}}_{\ell}}\left(\left(\pi_{q}^{z}\right)_{r^{\prime}} x_{q}+\left(\gamma_{q}^{z}\right)_{r^{\prime}} y_{q}\right)+\sum_{p \in \mathcal{P}^{r}}\left(\left(\delta^{p^{z}}\right)_{r^{\prime}} e_{r^{\prime}}^{p}\right. \\
& \left.+\left(\tau^{p^{z}}\right)_{r^{\prime}} \theta_{r^{\prime}}^{p}\right) \quad \forall z \in \mathcal{Z}_{r^{\prime}}, r^{\prime} \in \mathcal{S}_{\ell}^{1}, \ell \in \mathcal{L}_{r}
\end{aligned}
$$

$\lambda_{r^{\prime}} \in \mathbb{R} \quad \forall r^{\prime} \in \mathcal{S}_{\ell}^{1}, \ell \in \mathcal{L}_{r}$.

Note: $F_{r}\left(\hat{X}_{\sigma(r)}^{i}\right)$ for $r=1$ is a lower bound of the solution value $F_{r}^{\prime}($.) of model (21)-(33), where $\hat{X}_{\sigma(r)}^{i}=\emptyset$.

\subsection{SDP-ECSD algorithm}

\subsubsection{Forward scheme. Obtaining a feasible solution}

Let $\hat{X}^{i}=\left(\hat{x}_{g}, \hat{y}_{g}, \forall g \in \mathcal{G}\right)$ denote a feasible solution for the $r$ rooted subproblems (36)-(40) $\forall r \in \mathcal{R}^{E}$ obtained in the forward scheme at iteration, $i$, provided that $\epsilon_{r}^{p^{1}}$ and $\epsilon_{r}^{p^{2}}$ are small enough $\forall p \in \mathcal{P}^{r}, r \in \mathcal{R}^{E}$, see (41). Let also its related solution value $\hat{F}^{i}$ be computed as $\hat{F}^{i}=\sum_{g \in \mathcal{G}} w_{g}\left(a_{g} \hat{x}_{g}+c_{g} \hat{y}_{g}\right)$. On the other hand, let $i^{*}$ denote the iteration where the incumbent value has been obtained.

It is assumed that $\hat{X}^{i t e}$ is feasible if, besides satisfying the RN inherited constraints, it also does the following constraint,

$$
\frac{\hat{\epsilon}_{r}^{p^{1}}}{\overline{\bar{e}}^{p}} \leq \epsilon_{1} \text { and } \frac{\hat{\epsilon}_{r}^{p^{2}}}{\bar{\theta}^{p}} \leq \epsilon_{2} \quad \forall p \in \mathcal{P}_{g}, g \in \mathcal{G}_{t}, t \in \tilde{\mathcal{T}},
$$

where $\hat{\epsilon}_{r}^{p^{1}}$ and $\hat{\epsilon}_{r}^{p^{2}}$ are the violations of the ECSD bounds, and $\epsilon_{1}$ and $\epsilon_{2}$ are modeler-driven tolerances for still declaring feasibility. So, those violations can be expressed as follows,

$$
\begin{aligned}
& \hat{\epsilon}_{r}^{p^{1}}=\max \left\{\left(\sum_{\omega \in \Omega_{g}} w_{\omega} \hat{e}_{\omega}^{p}\right)-\bar{e}^{p}, 0\right\} \\
& \hat{\epsilon}_{r}^{p^{2}}=\max \left\{\left(\sum_{\omega \in \Omega_{g}} w_{\omega} \hat{\theta}_{\omega}^{p}\right)-\bar{\theta}^{p}, 0\right\},
\end{aligned}
$$

where $\hat{e}_{\omega}^{p}$ and $\hat{\theta}_{\omega}^{p}$ for $p \in \mathcal{P}_{g}, \omega \in \Omega_{g}, g \in \mathcal{G}_{t}, t \in \tilde{\mathcal{T}}$ are such that

$\hat{e}_{\omega}^{p}=\max \left\{\sum_{q \in \tilde{\mathcal{A}}_{\omega}}\left(a_{q} \hat{x}_{q}+b_{q} \hat{y}_{q}\right)-c^{p}, 0\right\}$,

$\hat{\theta}_{\omega}^{p}=1$ if $\hat{e}_{\omega}^{p}>0$ and, otherwise, 0.

The feasible solution $\hat{X}^{\text {ite }}$ (41) is assumed to improve the incumbent solution if the following testing is positive,

$$
\frac{\left(\hat{F}^{*} \hat{F}^{i t e}\right)}{\hat{F}^{*}}>\epsilon_{3},
$$

where $\epsilon_{3}$ is a positive tolerance.

\subsubsection{Backward scheme. Refining the EFV curves for improving the} variable $\lambda_{r^{\prime}}$ estimation

The mechanism in the backward scheme for refining the EFV variable $\lambda_{r^{\prime}}$ (39) at iteration ite of SDP-ECSD follows the steps presented in Escudero etal. (2015) but, now, considering the profiles in set $\mathcal{P}^{r}$, and the reference levels (35) in $\mathcal{Z}_{r^{\prime}}$, for $r^{\prime} \in \mathcal{S}_{\ell}^{1}, \ell \in$ $\mathcal{L}_{r}, r \in \mathcal{R}^{e}, e \in \mathcal{E} \backslash\{E\}$, instead of its expected aggregation vectors $\mu_{\ell}^{z}, \pi_{q}^{z}, \gamma_{q}^{z}$ and $\delta_{\ell}^{p^{z}}, \tau_{\ell}^{p^{z}}$ over the related $r^{\prime}$-vectors.

The backward scheme aims to refine the EFV curves in the $r$ rooted subproblems (36)-(40) by appending to the subproblems a set of multicuts (39) (as many as $\sum_{\ell \in \mathcal{L}_{r}}\left|\mathcal{S}_{\ell}^{1}\right|$ ). For that purpose, let nite ${ }^{3}$ be a modeler-driven parameter to denote the number of $r^{\prime}$ rooted subproblems to be solved, for $r^{\prime} \in \mathcal{S}_{\ell}^{1}, \ell \in \mathcal{L}_{r}$. The rhs of the constraint system (29)-(30) is the solution vector $\hat{X}_{\ell}^{i}$ (34) obtained in the forward scheme at iteration $i$, for $i=i t e-$ nite $^{3}+1, \cdots$, ite. As by-products of the solution of the $r^{\prime}$-rooted subproblems for each rhs, the reference levels (35) for $z=\left|\mathcal{Z}_{r^{\prime}}\right|+i$ are the elements of the new cuts (39) to be appended to the $r$-rooted subproblem (36)-(40). Constant $\left(\mu^{z}\right)_{r^{\prime}}$ can be computed as follows by using strong duality and Taylor's expansion,

$$
\begin{aligned}
\left(\mu^{z}\right)_{r^{\prime}} & =F_{r}\left(\hat{X}_{\ell}^{i}\right)-\sum_{q \in \tilde{\mathcal{A}}_{\ell}}\left(\left(\pi_{q}^{z}\right)_{r^{\prime}} \hat{X}_{q}^{i}+\left(\gamma_{q}^{z}\right)_{r^{\prime}} \hat{y}_{q}^{i}\right) \\
& +\sum_{p \in \mathcal{P}^{r}}\left(\left(\delta^{p^{z}}\right)_{r^{\prime}} \hat{e}_{r^{\prime}}^{p^{i}}+\left(\tau^{p^{z}}\right)_{r^{\prime}} \hat{\theta}_{r^{\prime}}^{p^{i}}\right) .
\end{aligned}
$$

Observe the high number of cuts that could be appended to the root node $r$-rooted subproblem (as many as nite ${ }^{3} \sum_{\ell \in \mathcal{L}_{r}}\left|\mathcal{S}_{\ell}^{1}\right|$ ). So, a mechanism should be considered for removing non-active cuts. A reasonable criterion is as follows: A cut (39) is removed from the subproblem provided that it has been non-active in the forward scheme of the last, say, nite ${ }^{1}$ consecutive iterations (including the current one, ite). The expression for declaring non-active the $z$ thbased cut, for $z \in \mathcal{Z}_{r^{\prime}}, r^{\prime} \in \mathcal{S}_{\ell}^{1}, \ell \in \mathcal{L}_{r}$, is as follows,

$$
\begin{aligned}
\lambda_{r^{\prime}}- & {\left[\left(\mu^{z}\right)_{r^{\prime}}+\sum_{q \in \tilde{\tilde{\mathcal{A}}}_{\ell}}\left(\left(\pi_{q}^{z}\right)_{r^{\prime}} \hat{x}_{q}^{i}+\left(\gamma_{q}^{z}\right)_{r^{\prime}} \hat{y}_{q}^{i}\right)\right.} \\
& \left.+\sum_{p \in \mathcal{P}^{r}}\left(\left(\delta^{p^{z}}\right)_{r^{\prime}} \hat{e}_{r^{\prime}}^{p^{i}}+\left(\tau^{p^{z}}\right)_{r^{\prime}} \hat{\theta}_{r^{\prime}}^{p^{i}}\right)\right]>\epsilon_{4} .
\end{aligned}
$$

where $\epsilon_{4}$ is a positive tolerance. Notice $\mathcal{P}^{r}=\mathcal{P}_{r}$ for $r=1$. Note: By construction, nite $e^{1}=0$ means that the cut-removing strategy is not used.

The SDP-ECSD matheuristic is formally presented in Algorithm 1.

In Step 1 the forward scheme is executed at the current iteration ite, such that the subproblem $F_{r}\left(\hat{X}_{\sigma(r)}^{i t e}\right)(36)-(40)$ for $r \in \mathcal{R}^{e}, e \in$ $\mathcal{E}$ is solved. Additionally, the EFV non-active cut testing (46) is performed. Notice that node $r=1$ has not any ancestor one. For iteration ite $>1$ the mechanism (46) for removing the non-active cuts (39) is used.

The algorithm's execution is stopped in Step 2 of iteration ite if the modeler-driven upper bound, say, mite on the number of iterations is reached, or the modeler-driven upper bound, say, nite ${ }^{2}$ on the number of consecutive iterations without incumbent solution improvement has been reached. Let also the modeler-driven indicator first be such that if it is equal to ' $Y^{\prime}$ then only the first feasible solution is required; otherwise, first $=^{\prime} N^{\prime}$.

In Step 3 the backward scheme generates a nite ${ }^{3}$-cardinality multicut (39) in current iteration ite for $r^{\prime} \in \mathcal{S}_{\ell}^{1}, \ell \in \mathcal{L}_{r}, r \in$ $\mathcal{R}^{e-1}, e \in \mathcal{E} \backslash\{1\}$. The solution vectors $\hat{X}_{\ell}^{i}$ (34) obtained in the forward scheme in the set of the previous nite ${ }^{3}$ iterations $\{i\}$ are to be used for generating the multicut. Notice that each of those solutions is used for fixing the rhs of the constraint system (29)-(30) in the related $r^{\prime}$-rooted subproblem (36)-(40) to be solved in the backward scheme in iteration ite. So, for nite $e^{3}=1$, only the multicut based on the solution $\hat{X}_{\ell}^{\text {ite }}$ is generated. The multicut is appended to the immediate ancestor $r$-rooted subproblem (36)-(40), for $r \in \mathcal{R}^{e-1}, e \in \mathcal{E} \backslash\{1\}$.

The meaning of the stopping indicators is as follows: stop1, the first feasible has been obtained for indicator first $=^{\prime} Y^{\prime}$; stop2, the maximum allowed number of iterations mite has been reached; and stop3, the maximum allowed number of iterations nite ${ }^{2}$ without incumbent solution improvement has been reached. 


\begin{abstract}
Algorithm 1 SDP-ECSD for the tolerances $\epsilon_{1}, \epsilon_{2}, \epsilon_{3}$ and $\epsilon_{4}$, the cut removing parameter nite ${ }^{1}$, the stopping parameters mite and nite ${ }^{2}$, the multicut generation parameter nite ${ }^{3}$, and the indicator first.
\end{abstract}

\section{Step 0: (Initialization)}

Set $\quad \mathcal{Z}_{r^{\prime}}:=\emptyset \forall r^{\prime} \in \mathcal{R}^{e}, e \in \mathcal{E}, \quad$ ite $:=1, i^{*}:=0, \quad$ nonact $:=0$, noimpr $:=0, e:=1$.

Step 1: (Forward scheme: On solving the lexicographically ordered subtree induced subproblems)

For $e=1, \cdots, E$ : For $r \in \mathcal{R}^{e}$ :

Solve subproblem $F_{r}\left(\hat{X}_{\sigma(r)}^{i t e}\right)(36)-(40)$.Output: $\hat{X}_{\ell}^{i t e}$ (34) $\forall \ell \in$ $\mathcal{L}_{\text {r }}$.

If ite $>1$ and $e<E$ and the EFV non-active cut testing (46) is negative for $z \in \mathcal{Z}_{r^{\prime}}, r^{\prime} \in \mathcal{S}_{\ell}^{1}, \ell \in \mathcal{L}_{r}$ then; nonact :=0; otherwise:

Update nonact $:=$ nonact +1 and if nonact $=$ nite $^{1}$ then reset $\mathcal{Z}_{r^{\prime}}:=\mathcal{Z}_{r^{\prime}} \backslash\{z\}$.

Enfor $r$. Enfor $e$.

Step 2: (Stopping criteria testing)

If the solution vector $\hat{X}^{\text {ite }}$ is not feasible (i.e., it does not satisfy (41)) then go to Step 2.1.

If ite $=1$ or the incumbent solution improvement testing (44) is positive then:

If first $=^{\prime} Y^{\prime}$ then stop $1, i^{*}:=i t e$, noimpr $=0$ and go to Step3.

Step 2.1:

If ite $\geq$ mite then stop2.

Update noimpr $:=$ noimpr +1 , if noimpr $=$ nite $^{2}$ then stop3.

Step 3: (Backward scheme: Approximating the EFV curves in the lexicographically ordered subtree induced subproblems)

For $e=E, \cdots, 2$ : For $r \in \mathcal{R}^{e-1}$ : For $\ell \in \mathcal{L}_{r}$ : For $r^{\prime} \in \mathcal{S}_{\ell}^{1}$ :

Set local vector $\mathcal{Z}^{\prime}:=\emptyset$.

For $i=\max \left\{\right.$ ite - nite $\left.e^{3}+1,1\right\}, \cdots$, ite:

Set $z^{\prime}:=\left|\mathcal{Z}_{r^{\prime}}\right|+i$ and update $\mathcal{Z}^{\prime}:=\mathcal{Z}^{\prime} \cup\left\{z^{\prime}\right\}$.

Solve subproblem $F_{r^{\prime}}\left(\hat{X}_{\sigma\left(r^{\prime}\right)}^{i}\right)(36)-(40)$.Output: The duals of the new $z^{\prime}$ th reference level (35).

Compute constant $\left(\mu^{z^{\prime}}\right)_{r^{\prime}}$ (35) so that the $z^{\prime}$ th EFV cut (39) is generated.

Append the new cut to the $r$-rooted subtree induced subproblem (36)-(40).

Enfor $i$.

Update $\mathcal{Z}_{r^{\prime}}:=\mathcal{Z}_{r^{\prime}} \cup \mathcal{Z}^{\prime}$.

Enfor $r^{\prime}$. Enfor $\ell$. Enfor $r$. Enfor $e$.

Step 4: (Iteration ending)

Update ite :=ite +1 and go to Step 1 .

Table 1

TSCP instances' dimensions.

\begin{tabular}{llllllllll}
\hline$I D$ & $|\mathcal{J} E|$ & $|\mathcal{J} S|$ & $|\mathcal{J}|$ & $|\mathcal{D}|$ & $|\mathcal{I} R|$ & $|\mathcal{I}|$ & $|\mathcal{R}|$ & $|\mathcal{F}|$ & $\left|H_{r}\right|$ \\
\hline M1 & 2 & 3 & 5 & 3 & 5 & 8 & 10 & 7 & 1 \\
M2 & 2 & 3 & 5 & 3 & 5 & 8 & 10 & 7 & 1 \\
M3 & 4 & 6 & 10 & 6 & 10 & 16 & 20 & 7 & 1 \\
M4 & 4 & 6 & 10 & 6 & 10 & 16 & 20 & 7 & 1 \\
M5 & 2 & 3 & 5 & 3 & 5 & 8 & 10 & 4 & 3 \\
M6 & 2 & 3 & 5 & 3 & 5 & 8 & 10 & 4 & 3 \\
M7 & 4 & 6 & 10 & 6 & 10 & 16 & 20 & 4 & 3 \\
M8 & 4 & 6 & 10 & 6 & 10 & 16 & 20 & 4 & 3 \\
L1 & 10 & 20 & 30 & 15 & 40 & 60 & 20 & 7 & 1 \\
L2 & 10 & 20 & 30 & 15 & 40 & 60 & 20 & 7 & 1 \\
L3 & 10 & 20 & 30 & 30 & 40 & 60 & 25 & 7 & 1 \\
L4 & 10 & 20 & 30 & 30 & 40 & 60 & 25 & 7 & 1 \\
\hline
\end{tabular}

\section{Computational experience}

\subsection{Introduction}

In this section the performance of the RN STSCP model (1)-(16), synthesized in model (17), is illustrated as well as the risk averse models TSD (18) and ECSD (19). Two testbeds sets are considered in the experiment with 8 instances the first one and 4 instances the other one. The main goal of the experiment consists of empirically proving that tactical supply chain planing problems require very large formulations even for small to medium instances as those in Testbed 1. In order to do so, computational complexity results for the RN measure are shown using both solution approaches, namely, the SDP-ECSD matheuristic and the plain use of the MIP solver CPLEX v12.5 (CPLEX optimizer user manual, 2013). It is shown that the former outperforms the latter in elapsed time, which reaches the imposed time limit $(2 \mathrm{~h})$ in 11 out of the 12 test instances. Based on those results, CPLEX is only used as the MIP solver in the computational testing of the risk averse measures for subproblems arising in the matheuristic. The second goal consists of illustrating the cost risk reduction of the ECSD and TSD measures as well as showing the violations of the ECSD risk reduction constraints by the TSD and RN solutions.

The experiment was conducted on a PC with a $2.5 \mathrm{GHz}$ dualcore Intel Core i5 processor, $8 \mathrm{~Gb}$ of RAM and the operating system was OS X 10.9. For plain use of CPLEX the optimality tolerance is set to 0.001. The parameters of the strategies for SDP-ECSD that have been used in the experiment are as follows: nite ${ }^{1}=0$, nite $e^{2}=$ 200 , nite $e^{3}=1$, first $=^{\prime} N^{\prime}$, mite $=200, \epsilon_{1}=10^{-6}, \epsilon_{2}=10^{-6}, \epsilon_{3}=$ 0 and $\epsilon_{4}$ is not used.

The reporting of the main results of our computational experience is organized as follows: Section 6.2 presents the two testbeds of randomly generated instances of the realistic tactical supply chain planning problem we have experimented with. Sections 6.3 and 6.4 report the results of using the matheuristic SDP-ECSD for solving the risk averse measures ECSD (19) and TSD (18), respectively. And Section 6.5 is devoted to the computational comparison of plain CPLEX and the matheuristic for solving the RN model (17).

\subsection{The test instances}

The instances in the experiment are identified as $M m, m=$ $1, \ldots, 8$ in Testbed 1 and $L m, m=1, \ldots, 4$ in (larger) Testbed 2 . Table 1 shows the instances' dimensions. The headings are as follows: $|\mathcal{J} E|$, number of end-products; $|\mathcal{J S}|$, number of subassemblies; $|\mathcal{J}|$, number of products; $\mathcal{D} S$, number of demand markets; $|\mathcal{I} R|$, number of raw materials; $|\mathcal{I}|$, number of components; $|\mathcal{R}|$, number of resources; $|F|$, number of segments in the piecewise linear concave supplying commitment cost function for each raw material; and $\left|\mathcal{H}_{r}\right|$, number of segments in the resource capacity expansion in set $R_{2}$ for instances $M 1$ to $M 4$ and $L 1$ to $L 4$ and sets $R^{e}$ for $e=1,2$ for instances M5 to M8.

It can be observed in Table 1 that instances M5 to M8 are, basically, as instances $M 1$ to $M 4$, but changing parameters in the objective function and the constraint system. Another important difference lies in the type of resources to consider. Instances M5 to $M 8$ allow resources of type $R^{e}$, for capacity expansion in each stage $e=1,2$, instead of type $R_{2}$ as in instances $M 1$ to $M 4$ and $L 1$ to $L 4$ that allow capacity expansion in each period along the time horizon. In fact, set $R^{e}$ allows in the experiment three segments in the resources capacity expansion in the set of nodes in the scenario tree related to the first period of concerned stage $e$, and that expansion can be used along the nodes until the end of the stage. On the contrary, set $R_{2}$ allows one segment in the resources capacity expansion at each node in the scenario tree, and that expansion 
Table 2

STSCP models' dimensions.

\begin{tabular}{|c|c|c|c|c|c|c|c|c|c|c|c|c|c|c|c|c|c|c|}
\hline \multirow[b]{2}{*}{$I D$} & \multicolumn{6}{|c|}{ Scenario tree } & \multicolumn{4}{|c|}{ RN model } & \multicolumn{4}{|c|}{ TSD model } & \multicolumn{4}{|c|}{ ECSD model } \\
\hline & Structure & $T$ & $t^{*}$ & $\left|\mathcal{G}_{t^{*}}\right|$ & $|\mathcal{G}|$ & $|\Omega|$ & $n c$ & $n 01$ & $n S 2$ & $m$ & $n c$ & $n 01$ & $n S 2$ & $m$ & $n c$ & n01 & $n S 2$ & $m$ \\
\hline M1 & $2^{4} 2^{4}$ & 8 & 5 & 16 & 255 & 128 & 9005 & 2560 & 35 & 15119 & 9261 & 2816 & 35 & 15635 & 9261 & 2816 & 35 & 15695 \\
\hline M2 & $2^{4} 2^{5}$ & 9 & 5 & 16 & 511 & 256 & 17965 & 5120 & 35 & 30223 & 18477 & 5632 & 35 & 31251 & 18477 & 5632 & 35 & 31311 \\
\hline M3 & $2^{4} 2^{4}$ & 8 & 5 & 16 & 255 & 128 & 17882 & 5120 & 70 & 30110 & 18138 & 5376 & 70 & 30626 & 18138 & 5376 & 70 & 30686 \\
\hline M4 & $2^{4} 2^{5}$ & 9 & 5 & 16 & 511 & 256 & 35674 & 10240 & 70 & 60190 & 36186 & 10752 & 70 & 61218 & 36186 & 10752 & 70 & 61278 \\
\hline M5 & $2^{4} 2^{4}$ & 8 & 5 & 16 & 255 & 128 & 9005 & 2900 & 20 & 15459 & 9261 & 3156 & 20 & 15975 & 9261 & 3156 & 20 & 16035 \\
\hline M6 & $2^{4} 2^{5}$ & 9 & 5 & 16 & 511 & 256 & 17965 & 5460 & 20 & 30563 & 18477 & 5972 & 20 & 31591 & 18477 & 5972 & 20 & 31651 \\
\hline M7 & $2^{4} 2^{4}$ & 8 & 5 & 16 & 255 & 128 & 17882 & 5800 & 40 & 30450 & 18138 & 6056 & 40 & 30966 & 18138 & 6056 & 40 & 31026 \\
\hline M8 & $2^{4} 2^{5}$ & 9 & 5 & 16 & 511 & 256 & 35674 & 10920 & 40 & 60530 & 36186 & 11432 & 40 & 61558 & 36186 & 11432 & 40 & 61618 \\
\hline L1 & $2^{4} 2^{4}$ & 8 & 5 & 16 & 255 & 128 & 56808 & 17870 & 280 & 89848 & 57064 & 18126 & 280 & 90364 & 57064 & 18126 & 280 & 90424 \\
\hline L2 & $2^{4} 2^{5}$ & 9 & 5 & 16 & 511 & 256 & 113256 & 35790 & 280 & 179576 & 113768 & 36302 & 280 & 180604 & 113768 & 36302 & 280 & 180664 \\
\hline L3 & $2^{4} 2^{4}$ & 8 & 5 & 16 & 255 & 128 & 72168 & 17875 & 280 & 98808 & 72424 & 18131 & 280 & 99324 & 72424 & 18131 & 280 & 99384 \\
\hline L4 & $2^{4} 2^{5}$ & 9 & 5 & 16 & 511 & 256 & 143976 & 35795 & 280 & 197496 & 144488 & 36307 & 280 & 198524 & 144488 & 36307 & 280 & 198584 \\
\hline
\end{tabular}


Table 3

ECSD model (19) solved with the SDP-ECSD matheuristic.

\begin{tabular}{|c|c|c|c|c|c|c|c|c|c|c|c|c|}
\hline ID & objval $_{E C S D}$ & objval $_{O p}$ & $t_{E C S D}$ & ite & nprob & $\operatorname{dev}_{E C S D} \%$ & $c^{1}$ & $\bar{e}^{1}$ & $\bar{\theta}^{1}$ & $c^{2}$ & $\bar{e}^{2}$ & $\bar{\theta}^{2}$ \\
\hline M1 & 576767 & 460675 & 1206 & 56 & 952 & 0.35 & 489634 & 24888 & 0.51 & 544038 & 10880 & 0.25 \\
\hline M2 & 604267 & 456308 & 2405 & 51 & 867 & -2.23 & 517328 & 25866 & 0.32 & 574809 & 18195 & 0.19 \\
\hline M3 & 169701 & 167102 & 179 & 25 & 425 & 0.23 & 177052 & 25994 & 0.76 & 196724 & 3934 & 0.25 \\
\hline M4 & 191791 & 190269 & 2005 & 49 & 833 & -0.07 & 189406 & 13553 & 0.76 & 210451 & 4209 & 0.25 \\
\hline M5 & 942335 & 887436 & 70 & 13 & 221 & 46.46 & 535116 & 42932 & 0.56 & 602740 & 16674 & 0.38 \\
\hline M6 & 686788 & 534198 & 2329 & 51 & 867 & 1.43 & 543194 & 27159 & 0.6 & 603549 & 19105 & 0.32 \\
\hline M7 & 1655257 & 1484588 & 1031 & 25 & 425 & 34.64 & 1260000 & 105000 & 0.83 & 1470000 & 52500 & 0.48 \\
\hline M8 & 1435342 & 1190273 & 10703 & 74 & 1258 & 8.67 & 1155000 & 105000 & 0.82 & 1365000 & 52500 & 0.43 \\
\hline L1 & 375127 & 364232 & 21065 & 200 & 3400 & 3.53 & 389681 & 41299 & 0.5 & 405091 & 10081 & 0.15 \\
\hline L2 & 215544 & 229286 & 25032 & 200 & 3400 & 0.14 & 302316 & 12092 & 0.4 & 335907 & 5374 & 0.15 \\
\hline L3 & 253152 & 246722 & 22103 & 200 & 3400 & 14.03 & 278247 & 58458 & 0.4 & 309164 & 14598 & 0.15 \\
\hline L4 & 431792 & 428498 & 19403 & 200 & 3400 & 6.92 & 544140 & 27207 & 0.51 & 604600 & 12092 & 0.13 \\
\hline
\end{tabular}

Table 4

TSD model (18) solved with the SDP-ECSD matheuristic.

\begin{tabular}{llllllllll}
\hline ID & objval $_{\text {TSD }}$ & $t_{\text {TSD }}$ & ite & nprob & $\operatorname{dev}_{\text {TSD }} \%$ & $v\left(\bar{e}_{\text {TSD }}^{1}\right) \%$ & $v\left(\bar{\theta}_{\text {TSD }}^{1}\right) \%$ & $v\left(\bar{e}_{\text {TSD }}^{2}\right) \%$ & $v\left(\bar{\theta}_{\text {TSD }}^{2}\right) \%$ \\
\hline M1 & 572235 & 2549 & 77 & 1309 & -0.44 & 73 & 0 & 74 & 50 \\
M2 & 605184 & 4004 & 72 & 1224 & -2.08 & 36 & 186 & 20 & 400 \\
M3 & 169290 & 761 & 70 & 1190 & -0.01 & 0 & 32 & 0 & 300 \\
M4 & 191001 & 5573 & 100 & 1700 & -0.49 & 0 & 32 & 0 & 300 \\
\hline
\end{tabular}

Table 5

RN model (17) solved with CPLEX and the SDP-ECSD matheuristic.

\begin{tabular}{|c|c|c|c|c|c|c|c|c|c|c|c|c|}
\hline \multirow[b]{2}{*}{ ID } & \multicolumn{3}{|l|}{ CPLEX } & \multicolumn{9}{|l|}{ SDP-ECSD } \\
\hline & objval $_{I P}$ & $t_{I P}$ & OG\% & objval $_{R N}$ & $t_{R N}$ & ite & nprob & GG\% & $v\left(\bar{e}_{R N}^{1}\right) \%$ & $v\left(\bar{\theta}_{R N}^{1}\right) \%$ & $v\left(\bar{e}_{R N}^{2}\right) \%$ & $v\left(\bar{\theta}_{R N}^{2}\right) \%$ \\
\hline M1 & 562705 & 3074 & 0.10 & 574783 & 12 & 15 & 255 & 2.15 & 102 & 0 & 127 & 50 \\
\hline M2 & 592726 & 7200 & 0.38 & 618020 & 15 & 15 & 255 & 4.27 & 86 & 74 & 40 & 67 \\
\hline M3 & 168066 & 7200 & 0.32 & 169308 & 38 & 18 & 306 & 0.74 & 0 & 15 & 44 & 50 \\
\hline M4 & 189810 & 7200 & 0.45 & 191934 & 65 & 24 & 408 & 1.12 & 131 & 32 & 165 & 200 \\
\hline M5 & 616644 & 7200 & 0.20 & 643412 & 113 & 20 & 340 & 4.34 & 153 & 0 & 24 & 0 \\
\hline M6 & 641666 & 7200 & 0.43 & 677103 & 351 & 25 & 425 & 5.52 & 137 & 0 & 53 & 0 \\
\hline M7 & 1207170 & 7200 & 0.04 & 1229357 & 638 & 35 & 595 & 1.84 & 57 & 0 & 0 & 200 \\
\hline M8 & 1245789 & 7200 & 0.45 & 1320940 & 1032 & 37 & 629 & 6.03 & 104 & 10 & 8 & 0 \\
\hline L1 & 358305 & 7200 & 0.62 & 362350 & 1409 & 76 & 1292 & 1.13 & 0 & 25 & 0 & 0 \\
\hline L2 & 212922 & 7200 & 1.14 & 215236 & 3884 & 72 & 1224 & 1.09 & 52 & 9 & 4 & 25 \\
\hline L3 & 218343 & 7200 & 0.72 & 221997 & 5032 & 200 & 3400 & 1.67 & 0 & 88 & 0 & 150 \\
\hline L4 & 398303 & 7200 & 0.28 & 403833 & 8055 & 200 & 3400 & 1.39 & 0 & 96 & 0 & 700 \\
\hline
\end{tabular}

can only be used in that node. Another important difference lies in the number of segments in the raw material supplying commitment cost function. All together it can be seen in Tables 3 and 5 the high impact in the objective function and computational effort that is required by using one or the other type of instances.

Table 2 presents the structure of the scenario tree for each instance as well as the dimensions of the three stochastic formulations. The set of periods, $\mathcal{T}$, has been split in two stages for the resource set $R^{e}, e=1,2$, and, in any case, for problem solving by the matheuristic SDP-ECSD. The first column of the table is the identifier of the instance, and the second one gives the predefined structure $A_{1}^{B_{1}} A_{2}^{B_{2}}$ of the scenario tree, where $A_{i}$ denotes the number of children each node in stage $i$ has and $B_{i}$ denotes the number of periods in stage $i$. The period subset $\tilde{\mathcal{T}}$ is a singleton and $t^{*} \in \tilde{\mathcal{T}}$, where $t^{*}$ is the period defining the groups of scenarios for cost risk reduction in the ECSD measure. The headings of the columns for the dimensions of the models are as follows: $n c$, number of continuous variables; $n 01$, number of $0-1$ variables; $n S 2$, number of S2 variables; and $m$, number of constraints.

The risk reduction on the TSCP cost is to be performed by considering two profiles for testing each of the risk reduction measures, namely TSD (18) and ECSD (19). The first period of the second stage, $t^{*}$, has been chosen in ECSD model for deciding the set of scenario groups where the cost risk reduction is to be performed. So, the additional number of constraints with respect to
RN model (17) is $4|\Omega|+4\left|\mathcal{G}_{t^{*}}\right|$. And the additional number of the $e$-continuous variables (cost excess over the thresholds) and $0-1$ $\theta$-variables (probability of failure on satisfying the thresholds) is $2|\Omega|$ for each one.

On the other hand, the whole set of scenarios in the last period of the second stage (see Section 6.4) has been chosen in the TSD model for deciding where the cost risk reduction is to be performed. So, the additional number of constraints with respect to RN model (17) is $4|\Omega|+4$, and the additional number of the $e$ continuous and $0-1 \theta$-variables for each type is the same as for ECSD.

It is worth to point out that the TSD measure performs the cost risk reduction for the whole set of scenarios as an entity and, on the contrary, the ECSD one does it for each group of scenarios.

\subsection{Computational complexity of the ECSD measure}

The testing of the computational complexity for the ECSD measure (19) is carried out by using the two testbeds as for the RN measure (see Section 6.5). In this experiment the modeler-driven period $t^{*}$ has been chosen for deciding the scenario groups in $\mathcal{G}_{t^{*}}$ to perform the TSCP cost risk reduction.

The results for solving ECSD model (19) by matheuristic SDPECSD can be found in Table 3. It shows the three items of the profiles, namely $\left(c^{p}, \bar{e}^{p}, \bar{\theta}^{p}\right)$, for $p=1,2$ in the ECSD measure for 
each scenario group $g$ in set $\mathcal{G}_{t^{*}}$. The maximum cost allowed $\bar{c}_{g}$ has been set up to a big enough value. Some other headings are as follows: objval $l_{E C S D}$ and $t_{E C S D}$, ESCD (incumbent) value and related elapsed time (in seconds); and objval ${ }_{O p}$, operational TSCP cost that results by subtracting the supplying cost $\sum_{i \in \mathcal{I} R} s c_{i}$ of the raw material cost from the ECSD value objval ${ }_{E C S D}(1)$. Notice that the raw material cost is due to the commitment made at the beginning of the time horizon for its supplying along the time horizon. (So, it is assumed that the modeler-driven risk reduction is only performed on the operational cost). The other headings are as follows: $\operatorname{dev}_{E C S D} \%$, deviation of the ESCD cost from the RN one (see Table 5), expressed as $\operatorname{dev}_{E C S D} \%=\left(\right.$ objval $_{E C S D}-$ ${ }_{o b j v a l}$ )/objval $l_{R N} \%$; ite, number of iterations that have been performed by the matheuristic; nprob, number of MIP subproblems (21)-(33) that have been solved by the whole set of iterations; and the last block of columns in Table 3 gives the threshold $c^{p}$, and the bounds $\bar{e}^{p}$ and $\bar{\theta}^{p}$ for $p=1,2$ that have been considered in the model.

Notice the deterioration $\operatorname{dev}_{E C S D} \%$ of the expected TSCP total cost in the ECSD model (while satisfying the risk reduction strategy in the operational cost along the time horizon) with respect to the expected TSCP total cost in the RN model. It is usually positive, in fact, it is $14.03 \%$ in instance $L 3,34.64 \%$ in $M 7$ and $46.46 \%$ in M5. However, sometimes it is negative, in fact, it is $-2.33 \%$ M2 and $-0.07 \%$ in $M 4$. Those latter results could be due to the heuristic character of the approach, but there is also another important reason that is explained at the end of Section 6.5. Observe also in the table the high computational effort (measured in the elapsed time) that is required to obtain the ECSD solution.

\subsection{Computational results for TSD risk averse measure}

The testing of the computational complexity of SDP-ECSD for the TSD measure (18) is carried out by using the instances M1 to M4. As said above, the cost risk reduction is performed on the same set of profiles as considered for the ECSD measure in Section 6.3. The results can be found in Table 4. The first block of headings is as follows: objval ${ }_{T S D}$ and $t_{T S D}$, TSD value (for the chosen group of scenarios, i.e., set $\Omega$ ) and related elapsed time (in seconds); ite, number of iterations; nprob, number of solved MIP subproblems; and $\operatorname{dev}_{T S D} \%$, deviation of the TSD value from the RN value (see Table 5), expressed as $\operatorname{dev}_{T S D} \%=\left(\right.$ objval $_{T S D}-$ objval $\left._{R N}\right) /$ objval $_{R N} \%$.

Notice that TSD model (18) is less restrictive than ECSD model (19) for a case as ours where the parameters of the profiles are exactly the same for both models. Observe that the TSD measure satisfies the cost risk reduction bounds for the whole set of scenarios in a given period (in our case, the last one in the time horizon). However, it does not imply, at all, that those bounds are satisfied by each of the groups of scenarios that are considered for the ECSD measure. See in Table 4 the violation of the cost risk reduction ECSD bounds incurred by the TSD solution. The last block of columns reports the expected violations $v\left(\bar{e}_{T S D}^{p}\right)(47)$ and $v\left(\bar{\theta}_{T S D}^{p}\right)$ (48) of the ECSD bounds $\bar{e}^{p}$ and $\bar{\theta}^{p}$ for $p=1,2$. Let $\hat{x}_{q}$ and $\hat{y}_{q}$ denote the values of the vectors of variables $x_{q}$ and $y_{q}$ in the solution of the TSD model (18).

$$
\begin{aligned}
v\left(\bar{e}_{T S D}^{p}\right) & =\frac{v\left(e_{T S D}^{p}\right)}{\bar{e}^{p}} \% \text { where } \\
v\left(e_{T S D}^{p}\right) & =\sum_{g \in \mathcal{G}_{t^{*}}} w_{g}\left(\sum_{\omega \in \Omega_{g}} w_{\omega} \hat{e}_{\omega}^{p}-\bar{e}^{p}\right)_{+} \text {and } \hat{e}_{\omega}^{p} \\
& =\left(\sum_{q \in \tilde{\mathcal{A}}_{\omega}}\left(a_{q} \hat{x}_{q}+b_{q} \hat{y}_{q}\right)-c^{p}\right)_{+}
\end{aligned}
$$

and

$$
\begin{aligned}
v\left(\bar{\theta}_{T S D}^{p}\right) & =\frac{v\left(\theta_{T S D}^{p}\right)}{\bar{\theta}^{p}} \% \text { where } v\left(\theta_{T S D}^{p}\right) \\
& \left.=\sum_{g \in \mathcal{G}_{t^{*}}} w_{g}\left(\sum_{\omega \in \Omega_{g}} w_{\omega} \hat{\theta}_{\omega}^{p}\right)-\bar{\theta}^{p}\right)_{+},
\end{aligned}
$$

where $(.)_{+} \equiv \max \{., 0\}$. Observe in Table 4 that, generally, those violations are high, although they are equal to zero for some instances.

Notice that the elapsed time required for providing the TSD solution is one to two orders of magnitude higher than the time required for the RN solution, see Table 5 . On the other hand, although the ECSD model (19) has more constraints than the TSD model (18), the latter is more difficult to solve than the former by our SDP-ECSD algorithm. The methodology distributes the cost risk reduction bounds $\bar{e}^{p}$ - and $\bar{\theta}^{p}$ in a subproblem among its immediate successor ones at any iteration. So, SDP-ECSD is not an ideal scheme for risk reduction measures where those bounds have to be distributed among many subproblems. However, since the number of successor subproblems for each scenario group with a oneto-one correspondence with the root nodes in $\mathcal{G}_{t^{*}}$ is smaller than $|\Omega|$, then, by construction, matheuristic SDP-ECSD is more efficient for ECSD with $t^{*}>1$ than for TSD with $t^{*}=1$.

\subsection{Computational complexity for RN measure}

In this section the performance results of the plain use of CPLEX and SDP-ECSD are reported for solving the RN model (17) in the two testbeds whose dimensions have been given in Table 1 . The results are shown in Table 5. The first column refers again to the identifier of the instance. The following three columns reports the CPLEX results, where objval ${ }_{I P}$ is the RN value, $t_{I P}$ is the elapsed time (in seconds) to obtain it, and OG\% is its optimality gap (in\%). The smallest instance M1 gives a solution that satisfies the optimality tolerance but requires 51 minutes (approx.), mainly due to the S2 variables in the raw material piecewise linear supplying commitment cost functions. The optimization of the other instances reaches the allowed time $(2 \mathrm{~h})$ without proving the $0.1 \%$ optimality of the solution.

Another block of columns in Table 5 reports the SDP-ECSD results. The headings are as follows: objval $l_{R N}$ and $t_{R N}$, RN solution value and related elapsed time (in seconds); ite, number of iterations; nprob, total number of MIP subproblems solved; and GG\%, goodness gap, i.e., the deviation of the solution value obtained by the matheuristic from the value obtained by CPLEX, expressed as $G G \%=\left(\right.$ objval $_{R N}-$ objval $\left._{I P}\right) /$ objval $_{I P} \%$. First, we can observe that, generally, the elapsed time that is required by SDP-ECSD is very small and, on the other hand, the goodness gap of its RN value versus the one provided by CPLEX is not too-high (it goes form $0.74 \%$ to $6.03 \%$ ). Anyway, that gap could be smaller (at the price of higher elapsed time), provided that the CPLEX optimality tolerance used in the matheuristic is reduced from 0.05 to, say, 0.01 (while the number of iterations is being increased). Notice that the SDPECSD elapsed time of the instances included in Testbed 1 is one to two orders of magnitude smaller than the CPLEX time, but M8 where the former is $11.87 \%$ of the latter. Observe also that CPLEX reaches the time limit $(2 \mathrm{~h})$ in 11 out of the 12 instances we have experimented with, while the matheuristic requires more than $2 \mathrm{~h}$ in only one instance.

The final block of columns in Table 5 reports the violation of the ECSD risk reduction bounds $\bar{e}^{p}$ and $\bar{\theta}^{p}$ incurred by the RN value obtained by SDP-ECSD, being those bounds $v\left(\bar{e}_{R N}^{p}\right)(49)$ and $v\left(\bar{\theta}_{R N}^{p}\right)$ (50), respectively, for the profiles $p=1,2$. Let $\hat{x}_{q}$ and $\hat{y}_{q}$ denote the values of the vectors of variables $x_{q}$ and $y_{q}$ in the solution of the 
RN model (17), respectively.

$$
\begin{aligned}
v\left(\bar{e}_{R N}^{p}\right) & =\frac{v\left(e_{R N}^{p}\right)}{\bar{e}^{p}} \% \text { where } \\
v\left(e_{R N}^{p}\right) & =\sum_{g \in \mathcal{G}_{t^{*}}} w_{g}\left(\sum_{\omega \in \Omega_{g}} w_{\omega} \hat{e}_{\omega}^{p}-\bar{e}^{p}\right)_{+} \text {and } \hat{e}_{\omega}^{p} \\
& =\left(\sum_{q \in \tilde{\mathcal{A}}_{\omega}}\left(a_{q} \hat{x}_{q}+b_{q} \hat{y}_{q}\right)-c^{p}\right)_{+}
\end{aligned}
$$

and

$v\left(\bar{\theta}_{R N}^{p}\right)=\frac{v\left(\theta_{R N}^{p}\right)}{\bar{\theta}^{p}} \%$ where $v\left(\theta_{R N}^{p}\right)=\sum_{g \in \mathcal{G}_{t^{*}}} w_{g}\left(\sum_{\omega \in \Omega_{g}} w_{\omega} \hat{\theta}_{\omega}^{p}-\bar{\theta}^{p}\right)_{+}$.

Notice that the higher the violations, the higher the solution's risk of having a high TSCP cost in non-wanted scenarios. It can be confirmed in Table 5, where it is shown that there are $68.74 \%$ violations of the ECSD bounds (many of them with high values). Frequently, the price paid (see column $\operatorname{dev}_{E C S D} \%$ in Table 3 ) for satisfying those bounds is also high.

By comparing the solution values objval $E C S D$ and objval $l_{R N}$ in Tables 3 and 5, respectively, we can observe that the deterioration of the former with respect to the latter is sometimes negative. It could be due to the heuristic character of the approach. Notice that the elapsed time required by the matheuristic for solving the ECSD model (19) is much greater than the time required for solving the RN model (17). It confirms the common knowledge, namely the stochastic dominance strategies are computationally much harder than the RN one (requiring an elapsed time that is one to two orders of magnitude higher than the time required for obtaining the RN solution). It is due to the cross scenario constraints for satisfying the risk reduction measure. Notice that, probably, CPLEX could not even solve the ECSD model, since it could not do it for the RN one.

Observe also in Tables 4 and 5 that the TSD model (18) requires more iterations and elapsed time than the RN model (17), It confirms the above statement but, contrary to what we could expect, the deviation $d e v_{T S D}$ is negative. The negative values of $\operatorname{dev}_{E C S D}$ in Table 3 and $\operatorname{dev}_{T S D}$ in Table 4 could be due to the heuristic character of the proposed approach. However, there is another strong reason, specifically for the cases (very valid ones) where the risk reduction is not performed over the objective function as a whole, but only over the operational cost. Note that, in the experiment under consideration, the thresholds $c^{1}$ and $c^{2}$ are for the operational cost instead for the full cost function. The pressing for that risk reduction may induce a decrease on the raw material supplying commitment cost.

\section{Conclusions}

A modeling scheme for dynamic tactical supply chain planning under uncertainty is presented. It is modeled with full recourse in a multi-period scenario tree. The model is a stochastic version of a real-life multi-period deterministic mixed $0-1$ problem with large sized instances. It appears in the car, computer and domestic appliances assembly sectors, among others. In any case, the presence of $0-1$ variables is very frequent for modeling the either-or decisions as well as for implementing the risk averse measures under consideration. Another element in real-life problems is the piecewise linear concave and nondecreasing function of the supplying commitment raw material cost. Its modeling by using S2 sets introduce a high difficulty in the model solving mainly for large sized stochastic models. Additionally, the model considers what today is a policy in industry, namely no warehouses are encouraged for subassembly and end-product stocking, although some stocking is allowed in the plants; even the burden of raw material stocking is transferred to the suppliers.

A time consistent risk averse measure, so-called expected stochastic dominance (ECSD) has been introduced in this work for risk management in multi-period stochastic mixed 0-1 programs. It is a mixture, see model (19), of (time-inconsistent) two-stage measures existing in the literature, namely, first- and second-order stochastic dominance functionals. However, the measure has been expanded in this work to the multistage setting with the cost risk reduction being performed in modeler-driven groups of scenarios in chosen periods along the time horizon. And, so, the time consistency property of the new measure is guaranteed. The modeling of the proposed measure requires to consider groups of cross scenario constraints (as many groups as the number of nodes in the modeler-driven period subset), such that the nice structure of the scenario tree based constraints is destroyed. And, so, typical decomposition algorithms cannot handle in an affordable computational effort for solving large sized instances. A specialized version, so-called SDP-ECSD, of our matheuristic SDP (Escudero et al., 2015) has been proposed for problem solving.

A computational experiment has been carried out for assessing the validity of the risk reduction approach as opposed to the risk neutral ( $\mathrm{RN}$ ) one. The problem sizes that are considered in the experiment vary from medium to real-life dimensions and, so, they are up to 10 end-products, 20 subassemblies, 40 raw materials, 30 markets, 25 resources, 9 periods (related to a three month time horizon) and 256 scenarios in the tree. The RN model (17) has up to 200,000 (approx) constraints, 35000+ 0-1 variables, $140000+$ continuous variables and 280 S2 variables. On the other hand, the ECSD risk reduction measure considers two profiles for each of the modeler-driven 16 groups where the scenarios have been distributed. So, besides the dimensions that are inherited from the RN model, the ESCD model (19) has up to 1088 constraints, $5120-1$ variables and 512 continuous variables. The RN solution obtained by plain use of CPLEX has a good optimality gap (from $0.10 \%$ to $1.14 \%$ ), and the matheuristic SDP-ECSD has a reasonable goodness gap (from $0.74 \%$ to $6.03 \%$ ) while comparing it with CPLEX. The latter one is stopped when reaching the $2 \mathrm{~h}$ elapsed time limit in 11 out of 12 instances and, by contrary, the former requires a much smaller elapsed time (from 12 to 5032 seconds) in 11 out of 12 instances. On the other hand, the ECSD model is very much difficult to solve, due to the tight risk reduction policy for the TCSP operational cost. Notice that the RN model violates 32 out of the 48 bounds (each one by a high value) on the cost risk reduction profiles. The model was unaffordable for plain use of CPLEX. On the contrary, SDP-ECSD satisfies all of those constraints, but at a cost increase from $-2.3 \%$ (notice that it is a matheuristic approach) to $46.46 \%$ over the RN model, and the elapsed varies from $1 \mathrm{~min}$ to $7 \mathrm{~h}$. Although the conclusions to draw from those results are still provisional, it seems that the proposal made in this work is a potential good approach for dealing with the very difficult real STSCP problem.

As a future research we are planning to consider to jointly modeling the time-consistent ECSD measure (that has obvious advantages) and the time-inconsistent TSD measure. The reason is that the latter performs, in a direct way, cost risk reduction for intermediate periods of the time horizon. So, it could be very attractive for decision-makers while planning for a long enough time horizon. And, then, the SDP-ECSD algorithmic modifications are a challenge to work on.

\section{Acknowledgement}

The authors would like to thank to the two anonymous reviewers for their help on clarifying some concepts presented in the manuscript and strongly improving its presentation. 


\section{References}

Ahmed, S., 2006. Convexity and decomposition of mean-risk stochastic programs. Math. Program. Ser. B 106, 433-446.

Aldasoro, U., Escudero, L.F., Merino, M., Monge, J.F., Pérez, G., 2015. On parallelization of a stochastic dynamic programming algorithm for solving large-scale mixed 0-1 problems under uncertainty. TOP 23, 703-742.

Aldasoro, U., Escudero, L.F., Merino, M., Pérez, G., 2017. A parallel branch-and-fix coordination based matheuristic algorithm for solving large-scale multistage stochastic mixed 0-1 problems. Eur. J. Oper. Res. 258, 590-606.

Alem, D., Morabito, R., 2013. Risk averse two-stage stochastic programs in furniture plants. OR Spectrum 35 (4), 773-806.

Alonso-Ayuso, A., Carvallo, F., Escudero, L.F., Guignard-Spielberg, M., Pi, J., Puranmalka, R., Weinteraub, A., 2014. Medium range optimization of copper extraction planning under uncertainty in future copper prices. Eur. J. Oper. Res. 233, 711-726.

Alonso-Ayuso, A., Escudero, L.F., Garín, A., no, M.T.O., Pérez, G., 2003. An approach for strategic supply chain planning based on stochastic 0-1 programming. J. Global Optim. 26, 97-124.

Artzner, P., Delbaen, F., Eber, L., Health, D., 1999. Coherent measures of risk. Math. Finance 9, 203-228.

Artzner, P., Delbaen, F., Eber, L., Health, D., Ku, H., 2007. Coherent multi-period risk adjusted values and Bellman's principle. Ann. Oper. Res. 152, 5-22.

Asamov, T., Ruszczynski, A., 2015. Time-consistent approximations of risk-averse multistage stochatic optimization problems. Math. Program. Ser. A 153, 459-493.

Beale, E.M.L., Forrest, J.J.H., 1976. Global optimization using special ordered sets. Math. Program. 10, 52-69.

Birge, J.R., Louveaux, F.V., 2011. Introduction to Stochastic Programming, second ed. Springer.

Carœ, C.C., Schultz, R., 1999. Dual decomposition in stochastic integer programming. Oper. Res. Lett. 24, 37-45.

Cohen, M.A., Lee, H.L., 1989. Resource deployment analysis of global manufacturing and distribution networks. J. Manuf. Oper. Manag. 2, 81-104.

Cristobal, M.P., Escudero, L.F., Monge, J.F., 2009. On stochastic dynamic programming for solving large-scale tactical production planning problems. Comput. Oper. Res. 36, 2418-2428.

Dembo, R., 1991. Scenario immunization. Ann. Oper. Res. 30, 63-90.

Dupačová, J., Consigli, G., Wallace, S.W., 2000. Scenarios for multistage stochastic programs. Ann. Oper. Res. 100, 25-53.

Escudero, L.F., 1994. CMIT: a capacitated multilevel implosion tool for production planning. Eur. J. Oper. Res. 76, 511-528.

Escudero, L.F., 1995. Robust portfolios for mortgage-backed securities. In: Zenios, S.A. (Ed.), Quantitative Methods. AI and Supercomputers in Finance. Unicom, London, pp. 201-228.

Escudero, L.F., Garín, A., Merino, M., Pérez, G., 2016. On time stochastic dominance induced by mixed integer-linear recourse in multistage stochastic programs. Eur. J. Oper. Res. 249 (1), 164-176.

Escudero, L.F., Garín, A., Unzueta, A., 2016. Cluster lagrangean decomposition in multistage stochastic optimization. Comput. Oper. Res. 67, 48-62.

Escudero, L.F., Monge, J.F., Morales, D.R., 2015. An SDP approach for multi-period mixed $0-1$ linear programming models with stochastic dominance constraints for risk management. Comput. Oper. Res. 58, 32-40.

Escudero, L.F., Monge, J.F., Morales, D.R., Wang, J., 2013. Expected future value decomposition based bid price generation for large-scale network revenue management. Transp. Sci. 47, 181-197.

Esmaeilikia, M., 2013. A Tactical Supply Chain Planning Model with Multiple Flexibility Options. School of Aerospace, Mechanical and Manufacturing Engineering, RMIT University, Melbourne, Australia PhD thesis.

Esmaeilikia, M., Fahiminia, B., Sarkis, J., Govidan, K., Kumar, A., Mo, J., 2016. A tactical supply chain planning model with multiple flexibility options: an empirical evaluation. Ann. Oper. Res. 244, 429-454.

Esmaeilikia, M., Fahiminia, B., Sarkis, J., Govidan, K., Kumar, A., Mo, J., 2016. Tactical supply chain planning models with inherent flexibility: definition and review. Ann. Oper. Res. 244, 407-422.

Fahimnia, B., Tang, C.S., Davarzani, H., Sarkis, J., 2015. Quantitative models for managing supply chain risks: a review. Eur. J. Oper. Res. 247, 1-15.
Gade, D., Hackebeil, G., Ryan, S.M., Watson, J.-P., Wets, R.-B., Woodruff, D.L., 2016. Obtaining lower bounds from the progressive hedging algorithm for stochastic mixed-integer programs. Math. Program. Ser. B 157, 47-67.

Gaivoronski, A.A., Plug, G., 2005. Value-at-risk in portfolio optimization: properties and computational approach. J. Risk 7, 11-31.

Gollmer, R., Gotzes, U., Schultz, R., 2011. A note on second-order stochastic dominance constraints induced by mixed-integer linear recourse. Math. Program. Ser. B 126, 179-190.

Gollmer, R., Neise, F., Schultz, R., 2008. Stochastic programs with first-order stochastic dominance constraints induced by mixed-integer linear recourse. SIAM J. Optim. 19, 552-571.

Govindan, K., Fattahi, M., 2017. Investigating risk and robustness measures for supply chain network design under demand uncertainty: a case study of glass supply chain. Int. J. Prod. Econ. 183, 607-720.

Heckmann, I., Comes, T., Nickel, S., 2015. A critical review on supply chain risk-definition, measure and modeling. Omega 52, 119-132.

Heitsch, H., Römisch, W., 2009. Scenario tree reduction for multistage stochastic programs. Comput. Manag. Sci. 6, 117-133.

Ho, W., Zheng, T., Yildiz, H., Talluri, S., 2015. Supply chain risk management: a literature review. Int. J. Prod. Res. 53, 5031-5069.

Hoyland, K., Kaut, M., Wallace, S.W., 2003. A heuristic for moment-matching scenario generation. Comput. Optim. Appl. 24, 169-185.

Klein, W.K., 1986. Duality in Stochastic Linear and Dynamic Programming. Springer-Verlag.

Klein, W.K., van der Vlerk, M.H., 2006. Integrated chance constraints: reduced forms an algorithm. Comput. Manag. Sci. 3, 245-269.

Leövey, H., Römisch, W., 2015. Quasi-monte carlo methods for linear two-stage stochastic programming. Math. Program. Ser. B 151, 314-345.

CPLEX Optimizer User Manual, IBM ILOG., 2013. http://www-01.ibm.com/software/ integration/optimization/cplex-optimizer/.

Homem-de Mello, T., Pagnoncelli, B.K., 2016. Risk aversion in multistage stochastic programming: a modeling and algorithmic perspective. Eur. J. Oper. Res. 249, 188-199.

Nickel, S., Saldanha-da Gama, F., Ziegler, H.-P., 2012. A multistage stochastic supply network design problem with financial decisions and risk management. Omega 40, 511-524

Ogryczak, W., Ruszczyński, A., 1999. From stochastic dominance to mean-risk models: semi-deviations as risk measures. Eur. J. Oper. Res. 116, 33-50.

Pereira, M.V.F., Pinto, L.M.V.G., 1985. Stochastic optimization of a multireservoir hydroelectric system: a decomposition approach. Water Resour. Res. 21, 779-792.

Pflug, G.C., 2000. Some remarks on the value-at-risk and the conditional value-at-risk. In: Uryasev, S. (Ed.), Probabilistic Constrained Optimization. Methodology and Applications. Springer, pp. 272-281.

Pflug, G.Ch., Pichler, A., 2015. Dynamic generation of scenario trees. Comput. Optim. Appl. 62, 641-668.

Pflug, G.Ch., Pichler, A., 2015. Time consistent decisions and temporal decomposition of coherent risk functional. Math. Oper. Res. 41, 682-699.

Rockafellar, R.T., Uryasev, S., 2000. Optimization of conditional value-at-risk. J. Risk 2, 21-41.

Rudloff, B., Street, A., Valladão, D.M., 2014. Time consistency and risk averse dynamic decision models: definition, interpretation and practical consequences. Eur. J. Oper. Res. 234, 743-750.

Ruszczyński, A., 2010. Risk-averse dynamic programming for Markov decision processes. Math. Program. 125, 235-261.

Schultz, R., Tiedemann, S., 2003. Risk aversion via excess probabilities in stochastic programs with mixed-integer recourse. SIAM J. Optim. 14, 115-138.

Schultz, R., Tiedemann, S., 2006. Conditional value-at-risk in stochastic programs with mixed integer recourse. Math. Program. Ser. B 105, 365-386.

Shapiro, A., 2009. On a time consistency concept in risk averse multistage stochastic programming. Oper. Res. Lett. 37, 143-147.

Shapiro, J.F., 1993. Mathematical programming models and methods for production planning and scheduling. In: Graves, S.C., Kan, A.H.R., Zipkin, E. (Eds.), Logistics of Production and Inventory. North-Holland, pp. 371-443.

Zou, J., Ahmed, S., Sun, X.A., 2016. Nested Decomposition of Multistage Stochastic Integer Programs with Binary State Variables. School of Industrial and Systems Engineering, Georgia Institute of Technology, Atlanta, GA 30332, USA. Working paper 